Historic, Archive Document

Do not assume content reflects current scientific knowledge, policies, or practices. 




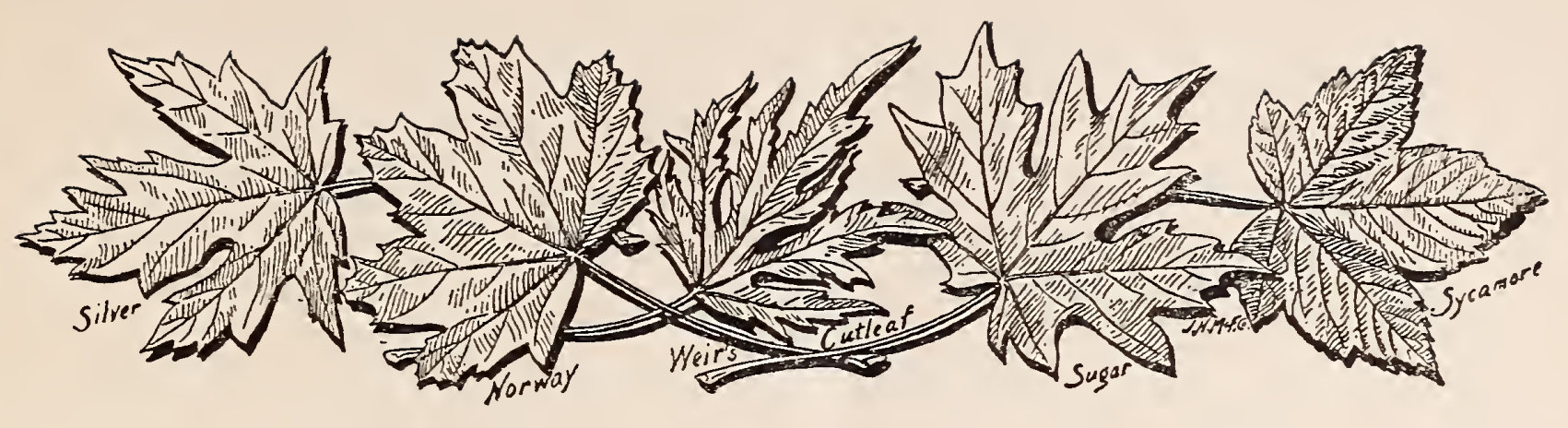

\section{CABLE ADDRESS}

\section{Suzuki, New York}

\section{Uyekigumi, Yokohama}

A. B. C. CODE-4th and 5th Editions and Western Union Used

NEW YORK, June, IgO4.

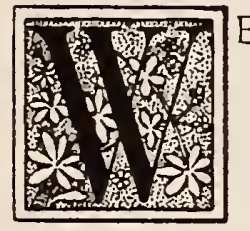

E take great pleasure in handing you our wholesale list, which we recommend to your careful examination.

Orders should reach us as soon as possible to secure full delivery, and not later than August I. Our Mr. B. Suzuki will be, as usual, in Japan to superintend the shipping.

\section{PRICES ARE FREE EX. CARS NEW YORK DUTY INCLUDED}

Terms, 50 per cent net cash on delivery, balance against three months' draft, or $2 \frac{1}{2}$ per cent discount on balance for cash within ten days. Orders from unknown customers accepted only when accompanied by cash for half the amount of order, or satisfactory reference.

Orders accepted subject to crop, and provided stock is unsold in Japan. It is understood, however, that we assume no responsibility for accidents or delays beyond our control.

Claims for errors and deficiencies must be made within ten days after delivery of goods. In case of any error on our part, it is mutually agreed between the purchaser and ourselves that we shall not, at any time, be held responsible for a greater amount than the original price of invoice.

San José Scale. All of our nursery stock is thoroughly fumigated in our own fumigator, which was built according to instructions received from Mr. Craw, State Entomologist of California.

\section{SUZUKI \& IIDA}




\section{Lily Bulbs}

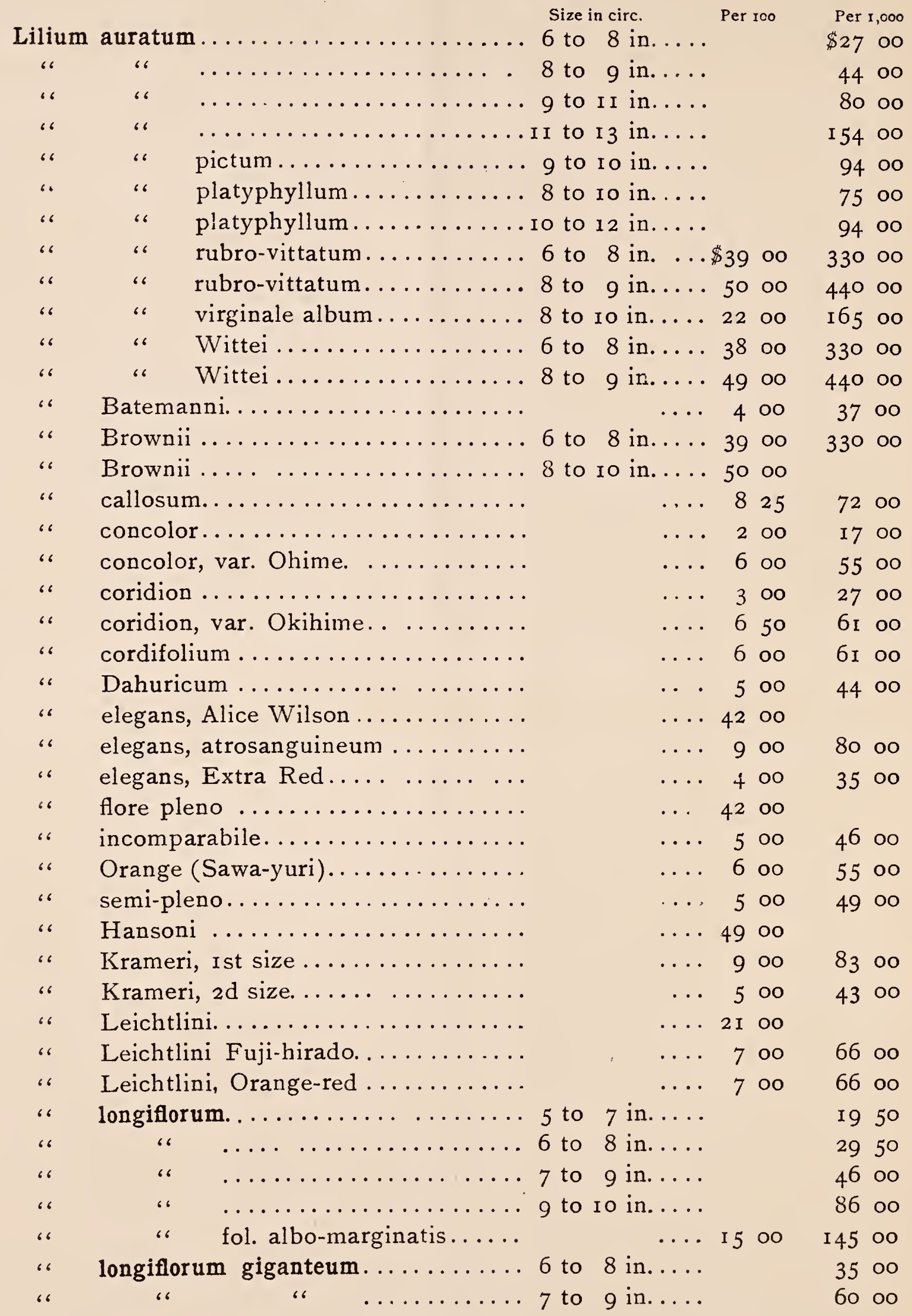


Size incirc. Per 100 Per $x, 000$ leaved variety) .......... $\$ 3$ to $8 \mathrm{in} . \ldots$. longiflorum multiflorum (narrow-

leaved variety)........... 7 to 9 in.... 5 I oo longiflorum multiflorum (narrow-

leaved variety) $\ldots \ldots \ldots \ldots \ldots$ to 9 to in..... medeoloides ............... ... rubellum ............... ... 8 oo speciosum album ........... 7 to 8 in.... "6 " $" \ldots \ldots \ldots \ldots \ldots$ to 9 in....

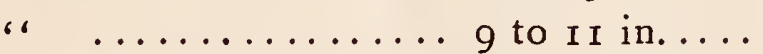

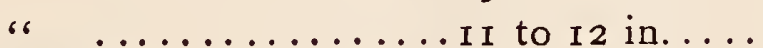
Krætzeri.......... 7 to 8 in.... Krætzeri.......... 8 to 9 in.... Krætzeri........... 9 to I I in.... Melpomene......... 8 to 9 in..... Melpomene......... 9 to so in.... rubrum...........6 6 to $8 \mathrm{in...6}$ $800 \quad 7500$ 5200 6000 I I O OO I 60 oo 6500 75 oo I 2000

47 oo 58 oo 2650 4100 6200 8300 tigrinum splendens.......... 8 to 9 in.... 4200 53 oo 48 oo

\section{Miscellaneous Bulbs and Roots}

Crinum Asiaticum (Spider Lily) . . . . . . . . . . . . \$

Erythronium Dens-Canis (Dog's-Tooth Violet)..........4 425

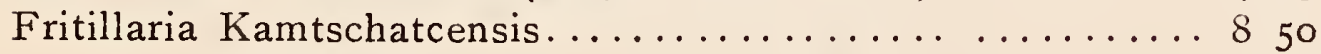

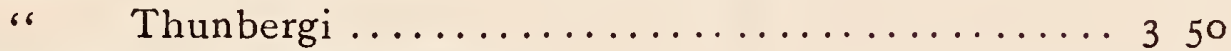

Hemerocallis aurantiaca major............... 6 oo

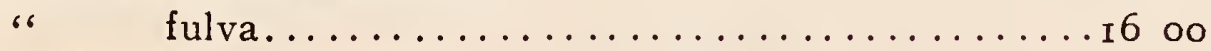

Iris Kæmpferi. Fifty best assorted varieties.......... I4 50

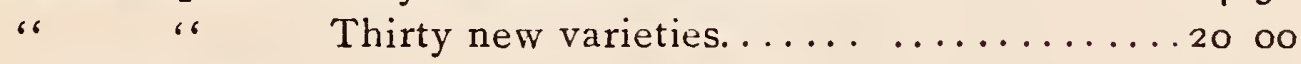

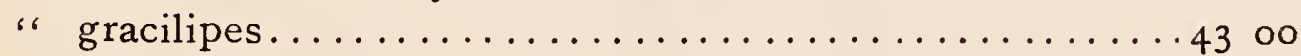

" Japonica............................ 35 . 50

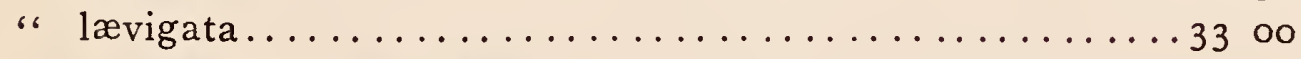

" lævigata fol. variegata. .................

" Sibirica orientalis...................23 oo

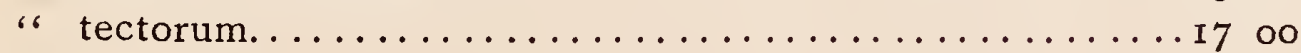

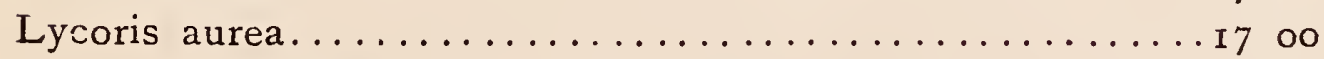

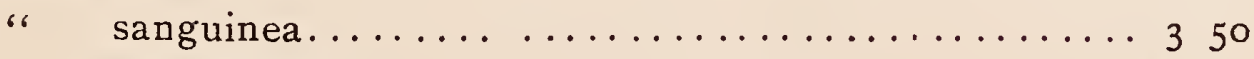

" squamigera...................... 17 oо

" "

Per 5,000

$\$$ I 300

38 oo

80 oo

3000

I 49 oo

14900

13400

9400 2900

\$4 00 
Nerine Japonica (Lycoris radiata). Scarlet.............. Per 100 Per 1,000 “ Japonica (Lycoris radiata). White. New variety....

Narcissus Tazetta (Japan Sacred Lily) ................ I I 50

“ Tazetta, Double ..................... I6 50

Pæonia, Herbaceous. Thirty best assorted varieties. The most beautiful collection ever offered........... 35 oo 330 oo

\section{Flowering Plants}

Azalea mollis Ki-renge. I to $\mathrm{I} / 2 \mathrm{ft} \ldots \ldots \ldots \ldots \ldots \ldots \ldots \ldots \ldots \ldots \ldots$

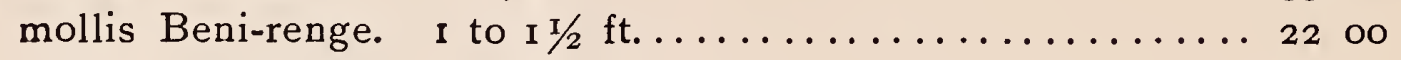

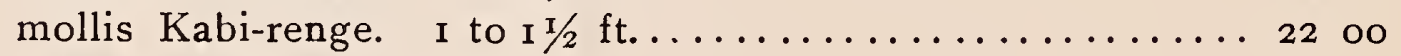

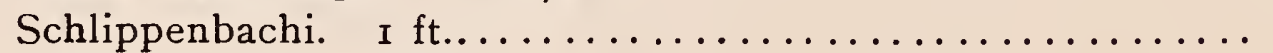

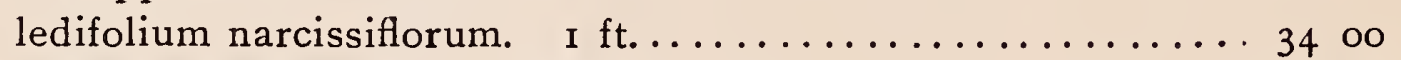

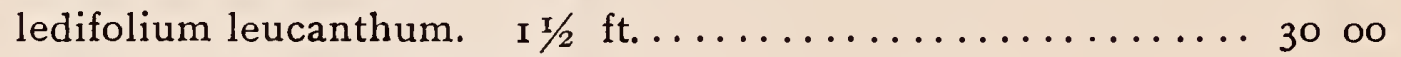

ledifolium Murasaki-Riukiu. I $\mathrm{ft} . \ldots \ldots \ldots \ldots \ldots \ldots$ I 8 oo

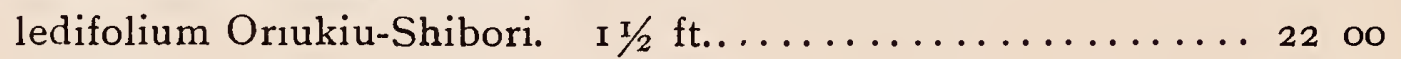

Akebono. $\quad \mathrm{ft} . \ldots \ldots \ldots \ldots \ldots \ldots \ldots \ldots \ldots \ldots \ldots$ I $8 \ldots \ldots$

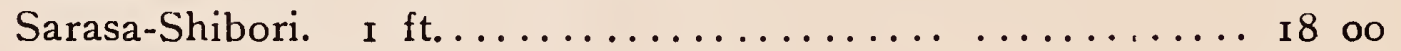

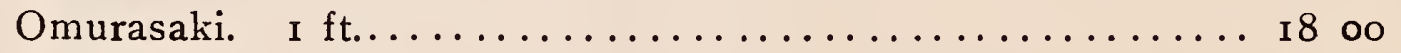

Tebotan. I ft........................ 40 oo

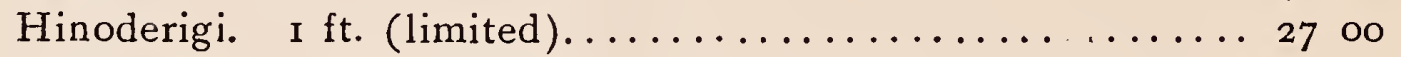

Hinoderigi. $2 \mathrm{ft}$ (limited) $\ldots \ldots \ldots \ldots \ldots \ldots \ldots \ldots \ldots \ldots$

Azuma Shibora. $\quad$ f $\mathrm{ft} \ldots \ldots \ldots \ldots \ldots \ldots \ldots \ldots$ I 8 oo

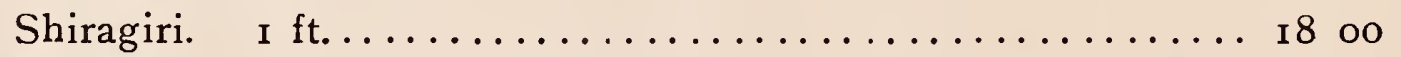

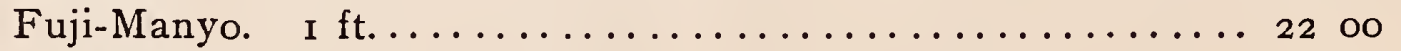

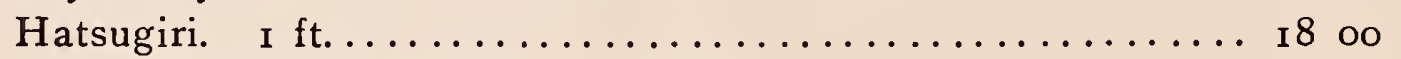

Indicum Tanima-no-yuki. I to $\mathrm{I} / 2 \mathrm{ft} \ldots \ldots \ldots \ldots \ldots \ldots \ldots$

Indicum Matsushima ...................... I 8 oo

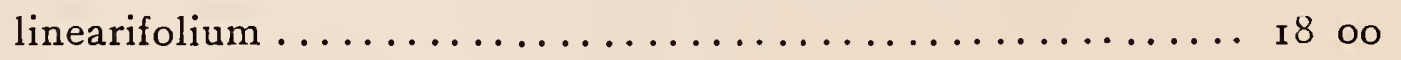

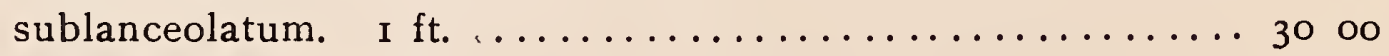

“ serpyllifolium. I ft..................... 6о о о

Camellia Japonica, Double. Fine assorted collection of 12 double

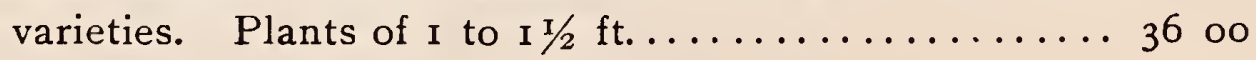

“ Japonica, Single. Four varieties............... 3 I 00

"Sasanqua, Mine-no-yuki. I to $2 \mathrm{ft} \ldots \ldots \ldots \ldots \ldots \ldots \ldots 45$ oo

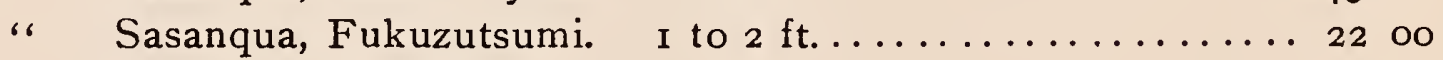

“Sasanqua, Mikuniko. I to $2 \mathrm{ft} \ldots \ldots \ldots \ldots \ldots \ldots \ldots \ldots \ldots$

“Sasanqua, Onigorono. I to $2 \mathrm{ft} . \ldots \ldots \ldots \ldots \ldots \ldots \ldots \ldots$

"Sasanqua, Azumanishiki. I to $2 \mathrm{ft} \ldots \ldots \ldots \ldots \ldots \ldots \ldots \ldots$

" theifera (Tea Plant). I to $2 \mathrm{ft} \ldots \ldots \ldots \ldots \ldots \ldots \ldots \ldots \ldots \ldots \ldots$

Hydrangea hortensis, var. Ajisai. I ft................ 22 oo

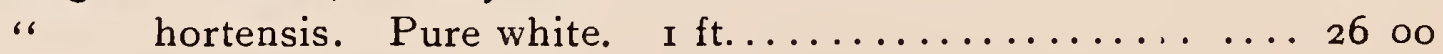

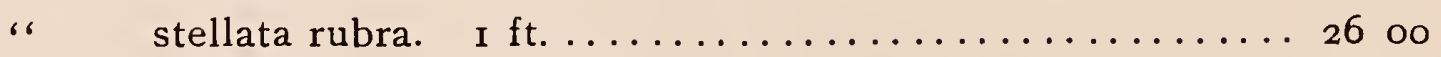

“ hortensis, var. Shirogaku. I ft............. 26 oо 
Hydrangea hortensis, var. Benigaku.

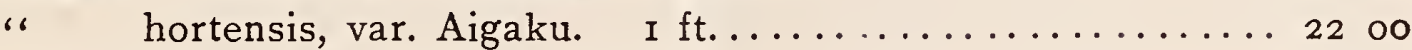

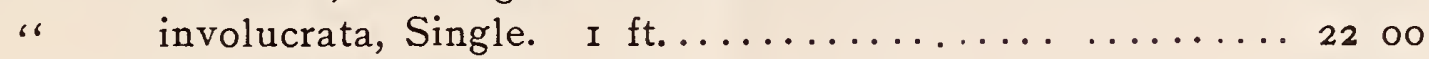

" involucrata, Double. I ft................. 45 o

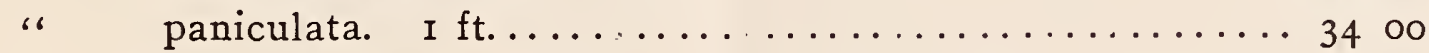

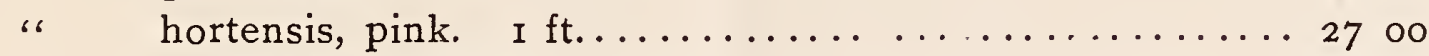

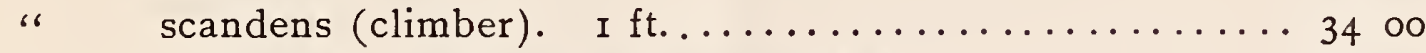

" petiolaris (Schizophragma hydrangeoides). I ft......... 4300

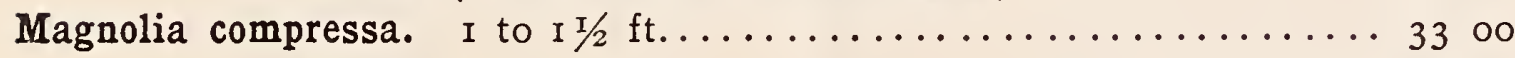

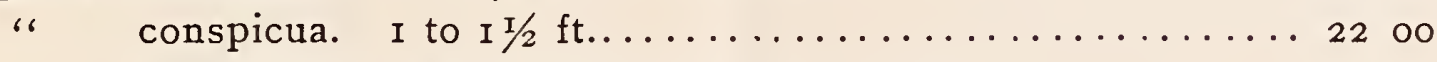

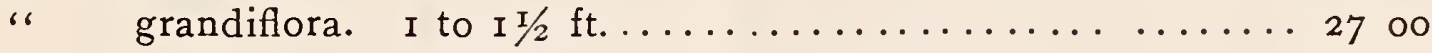

" hypoleuca. I to $2 \mathrm{ft} \ldots \ldots \ldots \ldots \ldots \ldots \ldots \ldots \ldots \ldots$ I 8 oo

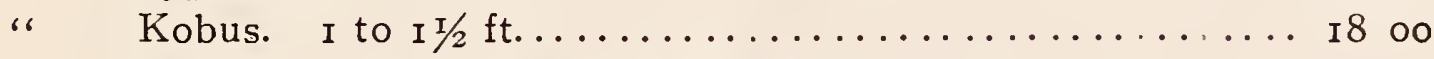

" (Michelia) champaca. I I/2 to $2 \mathrm{ft} . \ldots \ldots \ldots \ldots \ldots \ldots$

" (Michelia) longiflora. I $1 / 2$ to $2 \mathrm{ft} . \ldots \ldots \ldots \ldots \ldots \ldots \ldots$

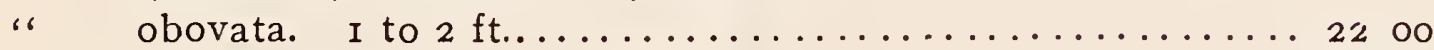

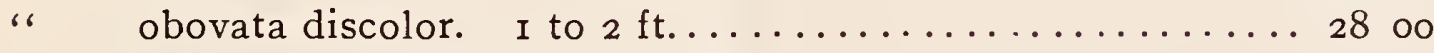

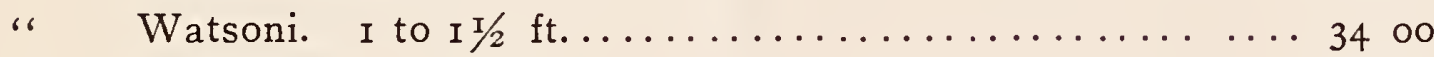

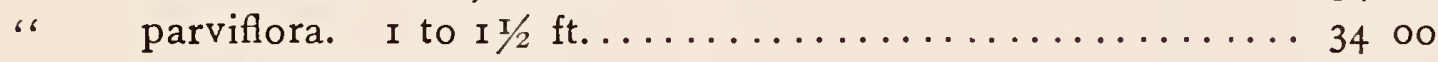

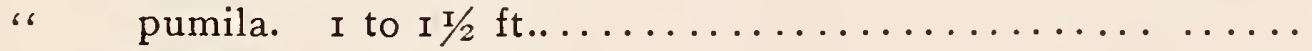

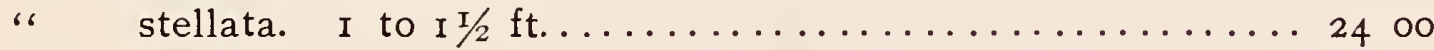

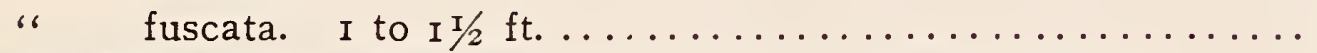

Pæonia Moutan (Tree Peonies), in 50 of the choicest varieties..... 4300

" " 5 to 6 years old, 7 to 8 flowers (supply limited) ....330 oo

Prunus Mume. Best assorted varieties of our choice pot-grown plants.. 22 oo

"Mume. Best assorted varieties of our choice open ground plants. I 3 oo

" Mume. Best persica (Japanese Peach). Best varieties...... 2200

" Pseudo-Cerasus (Japanese Cherry). Best vars., pot-grown.. 2200

" Pseudo-Cerasus (Japanese Cherry). Best vars., open ground. I3 oo

" Pseudo-Cerasus. Dwarfed, pot-grown.............66 o

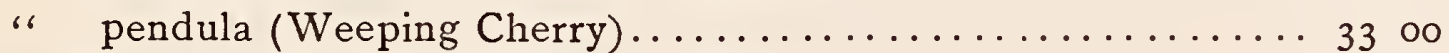

Punica granatum. Two flowering varieties $\ldots \ldots \ldots \ldots \ldots \ldots \ldots . \ldots 42$ o

Pyrus Japonica, Rose......................... 27 o

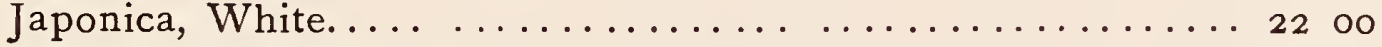

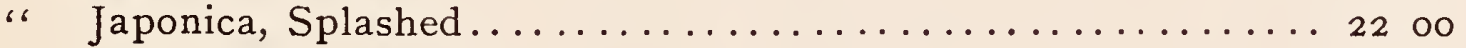

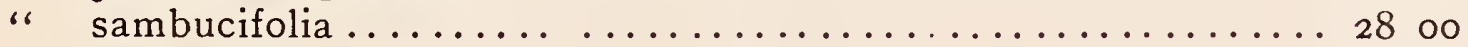

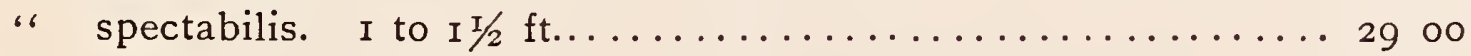

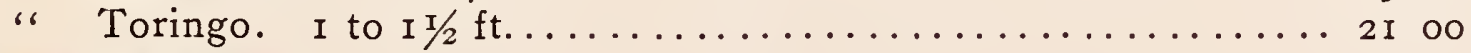

Roses, Japanese-

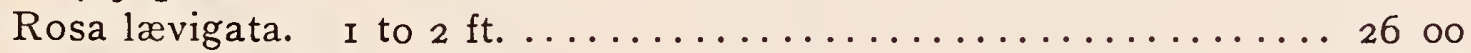

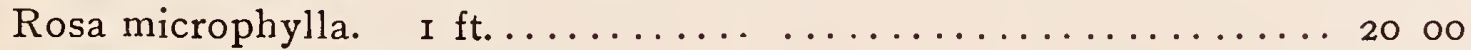

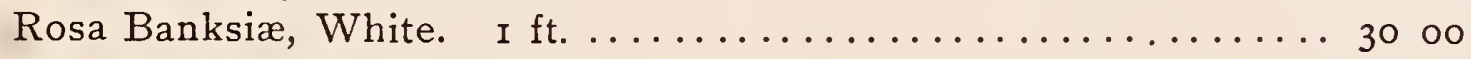

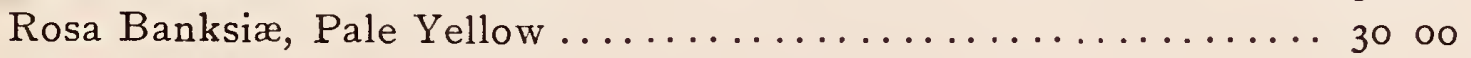

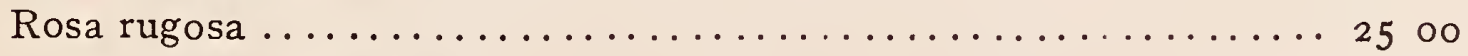

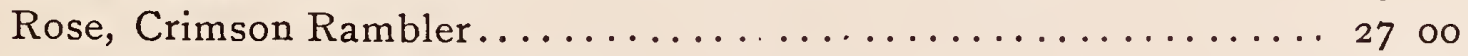

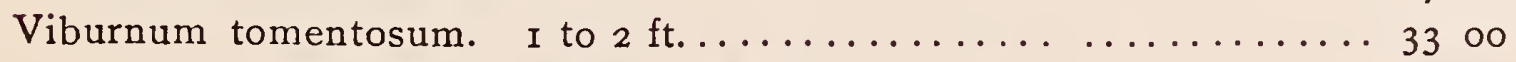

" tomentosum plicatum. I to $2 \mathrm{ft} . \ldots \ldots \ldots \ldots \ldots \ldots \ldots . \ldots . \ldots . \ldots$ 


\section{Deciduous Ornamental Trees and Shrubs}

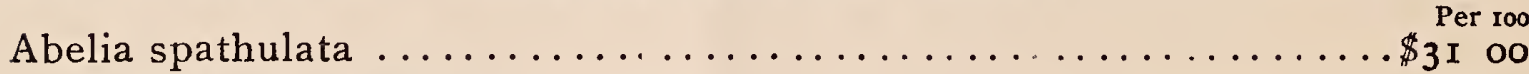

Acanthopanax aculeatum........................... 3 I oo

Acer polymorphum (Japanese Maples), in the following varieties :

" atropurpureum (Nomura)

"Oshiu-beni .

"Japonicum (Itaya)

" sanguineum (Seigen)

" roseum (Kagiri)

"versicolor (Oridono-nishiki)

"Osaka-zuki...

" atro-dissectum variegatum

" sanguineum (Chishio)

" atropurpureum dissectum, Deep Maroon .............

" atropurpureum dissectum, Green ...............

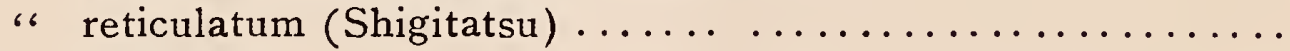

“Washino-o ..............................

“ Okushimo.............................

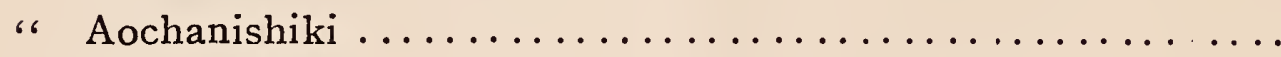

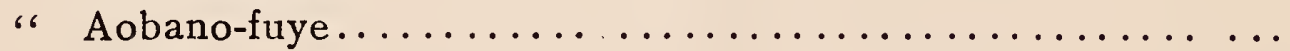

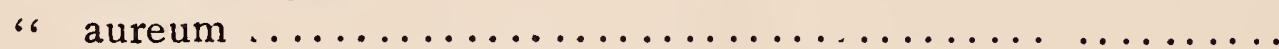

"scolopendifolium rubrum (Akashimenouchi) ...........

" scolopendifolium green (Aoshimenouchi) ..............

" atropurpureum variegatum $\ldots \ldots \ldots \ldots \ldots \ldots \ldots \ldots \ldots \ldots$

" Akikaze-nishiki ..........................

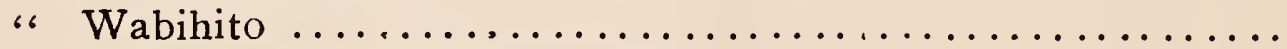

" rosa marginatis (Hikasayama) $\ldots \ldots \ldots \ldots \ldots \ldots \ldots \ldots \ldots$

" carpinifolium (Chidorikayede) $\ldots \ldots \ldots \ldots \ldots \ldots \ldots \ldots \ldots$

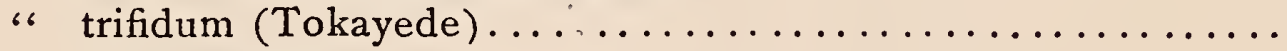

" Shishigashira..........................

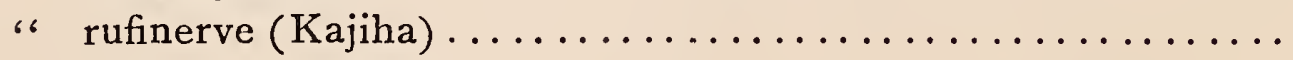

" Uriha-nishiki ...........................

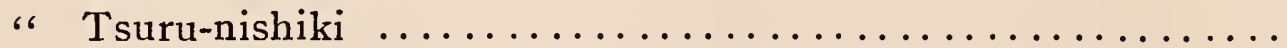

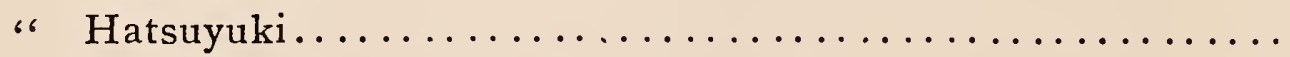

" Musatoriyama .........................

" pictum album (Tokiwanishiki)

" Japonicum filicifolium (Hauchiwa)

" Nishi Kigasane..$\ldots \ldots \ldots \ldots \ldots \ldots \ldots \ldots \ldots \ldots \ldots \ldots$

" pictum aureum (Hoshiyadori)

"Urime-nishiki

"Grafted Maples (Dantsugi). Five to seven kinds grafted on one

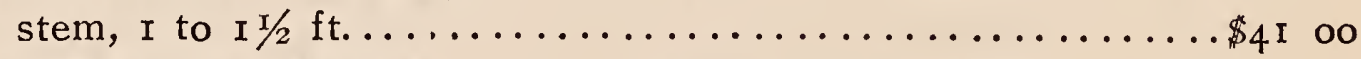

"Grafted Maples (Megane). Five to seven kinds grafted on one stem, I to $\mathrm{I} / 2 \mathrm{ft}$., in the form of an 8

I to $2 \mathrm{ft}$.

$\$ 33$

per 100.

2 to $3 \mathrm{ft}$.

$\$ 66$

per Ioo.

Per roo 


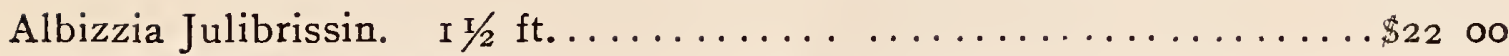

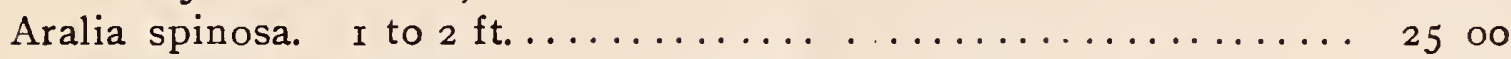

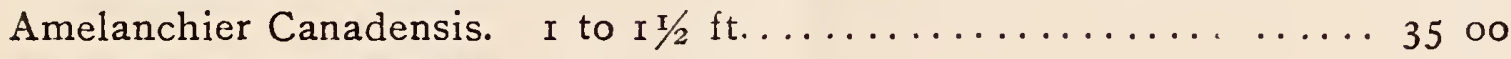

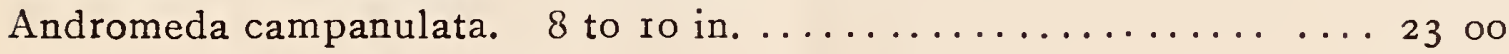

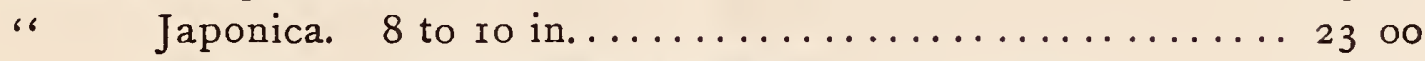

" Japonica. White marginal leaves. 8 to ro in........23 oo

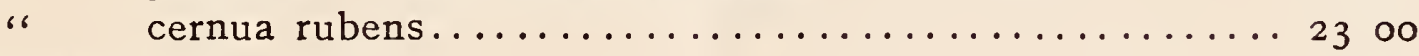

Berberis Japonica $\ldots \ldots \ldots \ldots \ldots \ldots \ldots \ldots \ldots \ldots \ldots \ldots \ldots \ldots \ldots \ldots$ r7

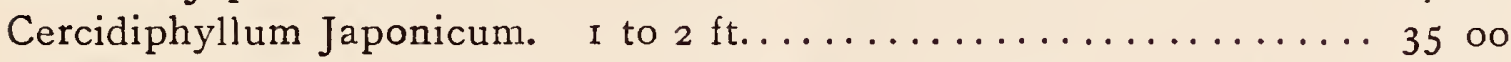

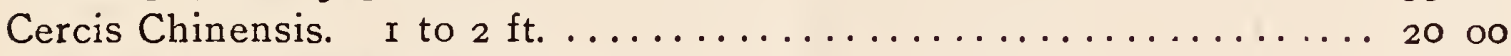

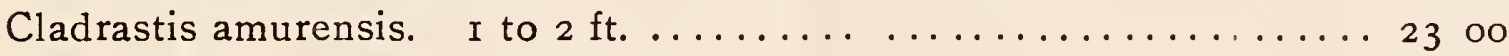

Clerodendrum squamatum. I to $\mathrm{r} / 2 \mathrm{ft} . \ldots \ldots \ldots \ldots \ldots \ldots \ldots \ldots$ rg oo

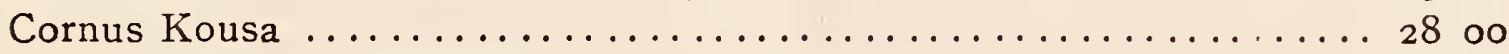

" Kousa, Variegated ...................... 33 o

" macrophylla............................ r9 oo

" macrophylla, Variegated .................... 44 oo

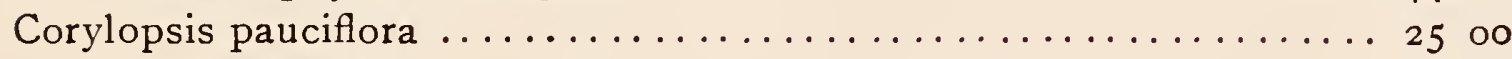

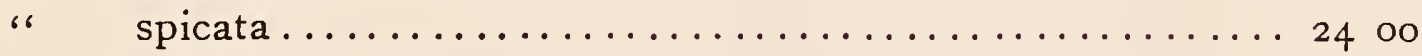

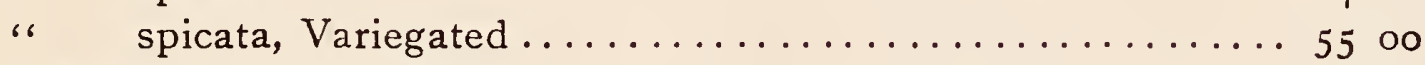

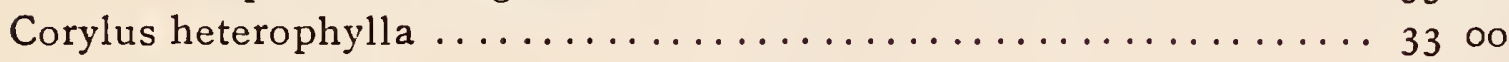

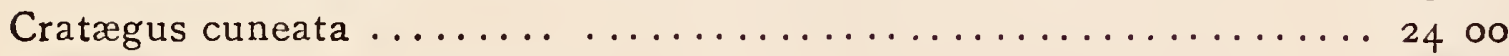

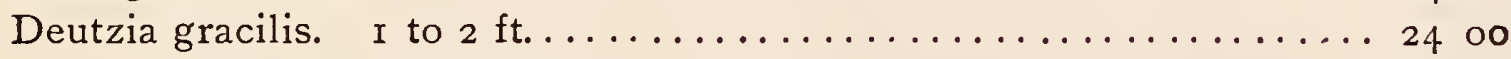

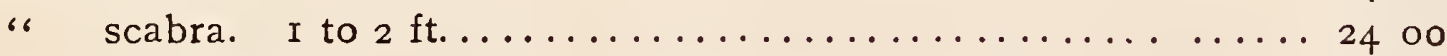

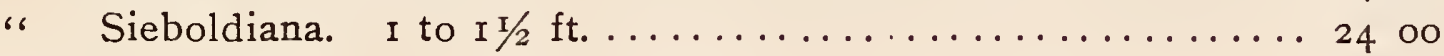

Diervilla grandiflora ........................ r

" hortensis. $\mathrm{I} / 2$ to $2 \mathrm{ft} \ldots \ldots \ldots \ldots \ldots \ldots \ldots \ldots \ldots \ldots \ldots \ldots \ldots \ldots$ r7

Enkianthus Japonicus ...................... r

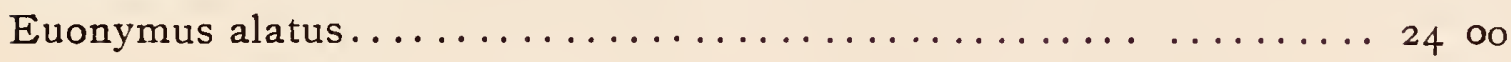

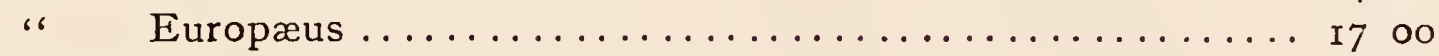

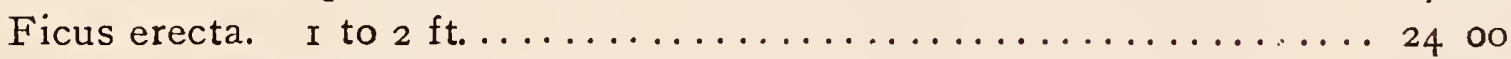

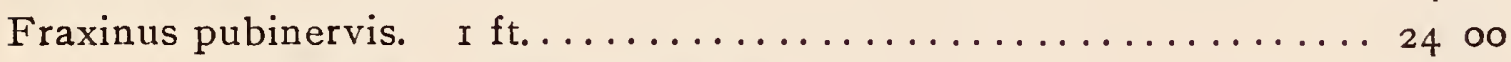

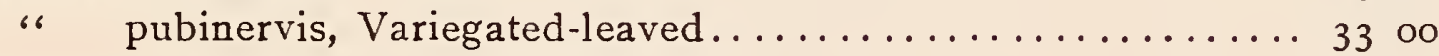

Hamamelis Japonica ........................... 24 o

Hypericum Chinense $\ldots \ldots \ldots \ldots \ldots \ldots \ldots \ldots \ldots \ldots \ldots \ldots \ldots$ r 4 oо

" patulum ......................... r 4 o

Ilex Sieboldi. Red berries. I to $\mathrm{I} / 2 \mathrm{ft} \ldots \ldots \ldots \ldots \ldots \ldots \ldots \ldots \ldots$

"Sieboldi. White berries. I to $\mathrm{r} / 2 \mathrm{ft} . \ldots \ldots \ldots \ldots \ldots \ldots \ldots \ldots \ldots \ldots$

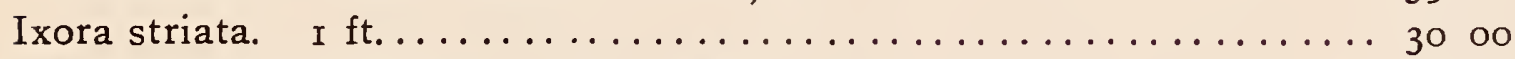

Jasminum Sieboldianum. $\mathrm{r}$ to $\mathrm{r} \mathrm{x} / \mathrm{ft} \ldots \ldots \ldots \ldots \ldots \ldots \ldots \ldots \ldots$ r 7 oo

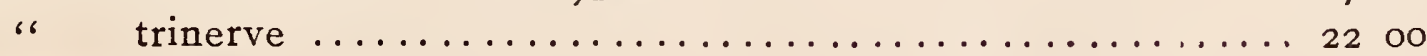

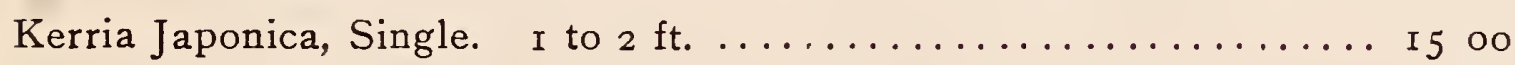

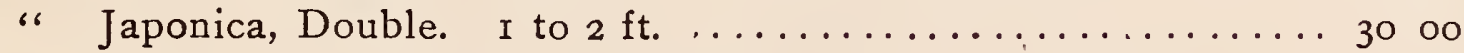

Lagerstrœmia Indica, Pink. I to $\mathrm{r} / 2 \mathrm{ft} \ldots \ldots \ldots \ldots \ldots \ldots \ldots \ldots \ldots$ I7 oo

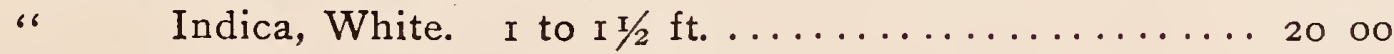

"Indica, Light Claret. I to $\mathrm{I} / 2 \mathrm{ft} . \ldots \ldots \ldots \ldots \ldots \ldots$ 
Lespedeza bicolor, Light Violet. I to $2 \mathrm{ft} . \ldots \ldots \ldots \ldots \ldots \ldots \ldots \ldots$

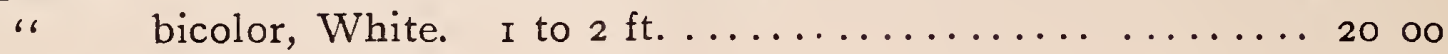

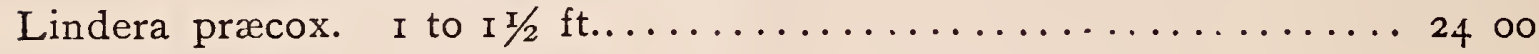

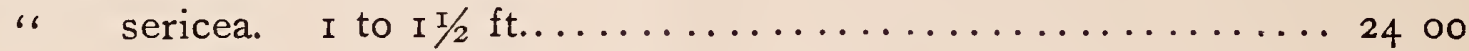

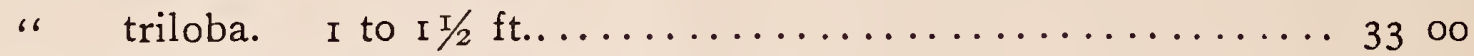

Rhodotypos kerrioides. I to $\mathrm{I} / 2 \mathrm{ft} \ldots \ldots \ldots \ldots \ldots \ldots \ldots \ldots \ldots \ldots \ldots$

Sophora Japonica. I ft...................... I7 oo

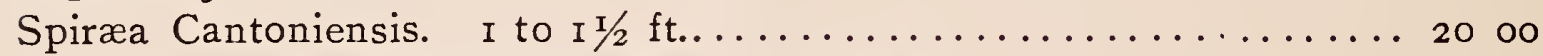

“ Japonica. White flowers. I to $\mathrm{I} / 2 \mathrm{ft} \ldots \ldots \ldots \ldots \ldots \ldots \ldots$ I7 0

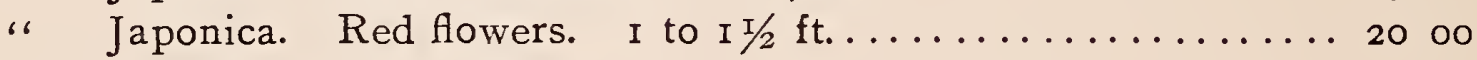

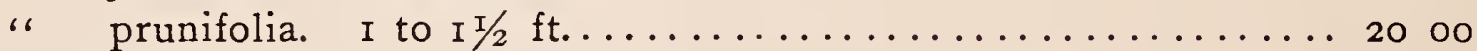

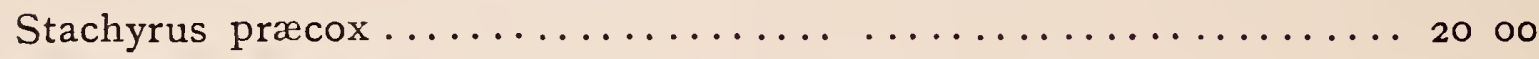

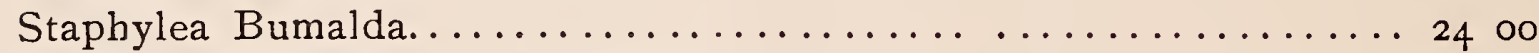

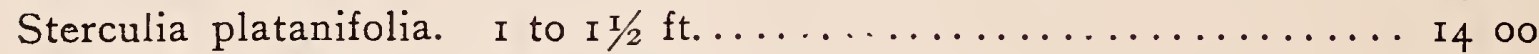

" platanifolia, Variegated. I to $\mathrm{I} I / 2 \mathrm{ft} \ldots \ldots \ldots \ldots \ldots \ldots \ldots 4400$

Stuartia Pseudo-Camellia. I to $\mathrm{I} / 2 \mathrm{ft} . \ldots \ldots \ldots \ldots \ldots \ldots \ldots \ldots \ldots \ldots$

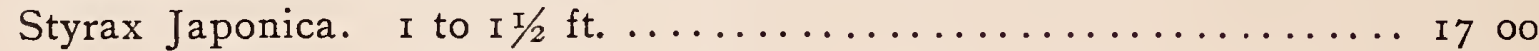

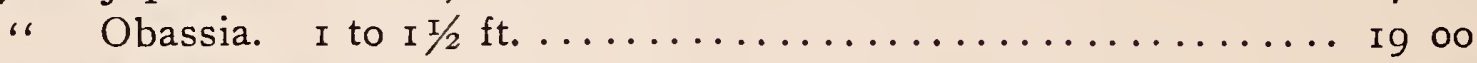

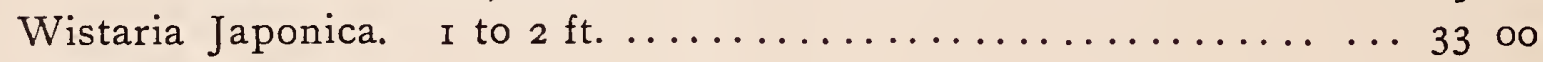

Zelkova Keaki............................. I9 oо

\section{Evergreen Ornamental Trees and Shrubs}

Ardisia crispa. Red berries. 8 to ro in............ Per 1o $\quad \begin{aligned} & \text { Per 100 } \\ & \$ 27\end{aligned} 50$

" crispa. White berries. 8 to ro in........... 2800

" crispa. White and red grafted on one plant. 8

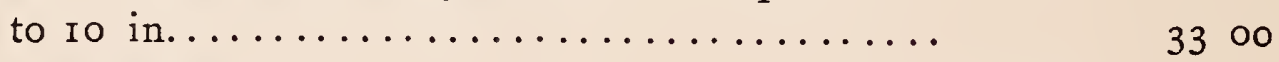

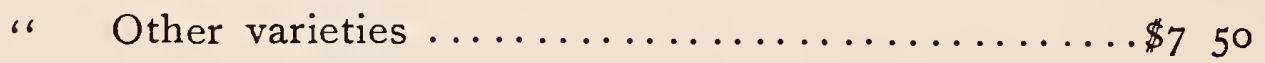

“ Japonica. 6 to 8 in. ................ 275

“Japonica, Other varieties............... 7 50

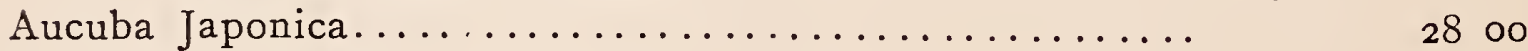

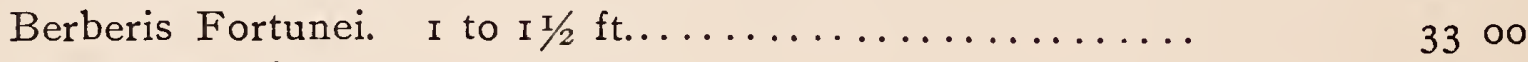

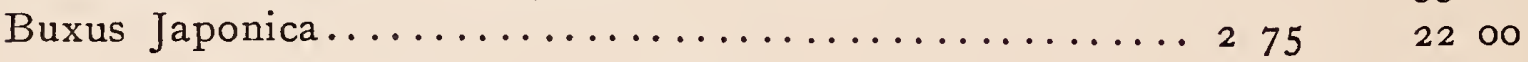

Cinnamomum Loureirii. I to $\mathrm{I} / 2 \mathrm{ft} . \ldots \ldots \ldots \ldots \ldots \ldots \ldots$ I5 00 .

" Loureirii. Variegated leaves. I to $\mathrm{I} / 2 \mathrm{ft} \ldots 275$

" sericeum. I to I $1 / 2 \mathrm{ft} . \ldots \ldots \ldots \ldots \ldots \ldots \ldots+\ldots \ldots$

Chloranthus brachystachys. I to $\mathrm{I} / 2 \mathrm{ft} \ldots \ldots \ldots \ldots \ldots \ldots$ oo

" brachystachys. Variegated leaves........ 625

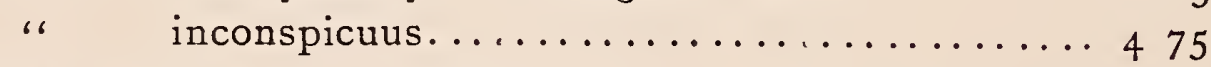

Cleyera Japonica. I to $\mathrm{I} / 2 \mathrm{ft} . \ldots \ldots \ldots \ldots \ldots \ldots \ldots \ldots \ldots$

"Japonica, White. Variegated leaves. I to I I $/ 2 \mathrm{ft} . .400$

“ Japonica, Yellow. Variegated leaves. I to I I/2 ft. . 4 oo

Damnacanthus Indicus. I to $\mathrm{I} / 2 \mathrm{ft} \ldots \ldots \ldots \ldots \ldots \ldots \ldots+\ldots$

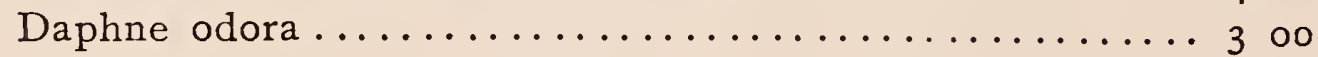


Daphne odora. White-margined leaves, white flower.....\$3

" odora. White-margined leaves, pink flower ..... 225

$\$ 2800$

Daphniphyllum glaucescens. I to I $1 / 2 \mathrm{ft} . \ldots \ldots \ldots \ldots \ldots 25$

I 900

" glaucescens. Variegated leaves. I to I $1 / 2 \mathrm{ft} .4$ oo

" macropodum. I to I $\mathrm{I} / 2 \mathrm{ft} \ldots \ldots \ldots \ldots \ldots 2$ oo

Dendropanax Japonicus. I to $I \mathrm{I} / 2 \mathrm{ft} \ldots \ldots \ldots \ldots \ldots 25$

Distylium racemosum. I to $\mathrm{I} / 2 \mathrm{ft} \ldots \ldots \ldots \ldots \ldots \ldots \ldots$

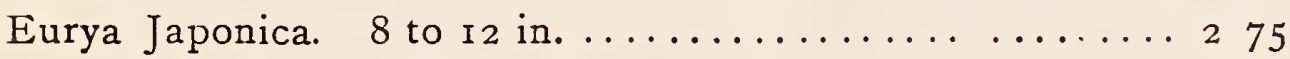

" Japonica. White, variegated. 8 to I 2 in........ 275

" Japonica. Yellow and other variegated. 8 to I 2 in. 275

Euscaphis staphyleoides. 8 to $\mathrm{r} 2$ in...................

" staphyleoides, Variegated. I to I $1 / 2 \mathrm{ft} . \ldots \ldots 650$

Fatsia Japonica. I to I $1 / 2 \mathrm{ft} . \ldots \ldots \ldots \ldots \ldots \ldots \ldots$

" Japonica, Variegated (Aralia Sieboldi). I to I I/2 ft. . 375

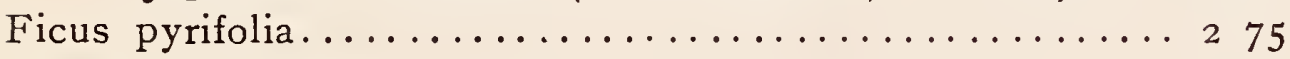

Gardenia grandiflora. I to I $\mathrm{x} / 2 \mathrm{ft} \ldots \ldots \ldots \ldots \ldots \ldots \ldots \ldots \ldots$

" grandiflora, Double. I to I $\mathrm{I} / 2 \mathrm{ft} \ldots \ldots \ldots \ldots \ldots 275$

" radicans. 6 to $8 \mathrm{in} \ldots \ldots \ldots \ldots \ldots \ldots \ldots \ldots$ I 75

" radicans, Double. 6 to 8 in............ 2 oo

" radicans, Double Yellow Variegated. Dwarf, 6 to 8 in.............................. oo

Ilex crenata. I to $\mathrm{I} / 2 \mathrm{ft} . \ldots \ldots \ldots \ldots \ldots \ldots \ldots \ldots \ldots \ldots$

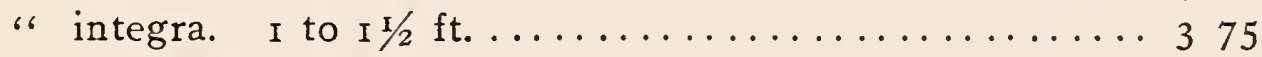

" integra, Variegated. I to $I / 2$ ft............. 525

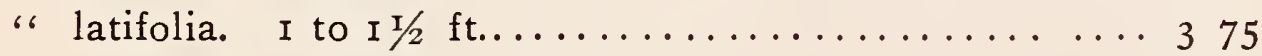

" latifolia, Variegated. I to I $1 / 2 \mathrm{ft}$. ......... 650

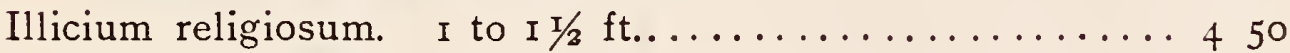

Ligustrum ciliatum. I I/2 to $2 \mathrm{ft} . \ldots \ldots \ldots \ldots \ldots \ldots \ldots$

" ciliatum, Variegated. I to I I/2 ft. ....... 225

$"$ Ibota. I to I $1 / 2 \mathrm{ft} \ldots \ldots \ldots \ldots \ldots \ldots \ldots \ldots \ldots$ I4 00

" Japonicum. I to I $1 / 2 \mathrm{ft} . \ldots \ldots \ldots \ldots \ldots \ldots \ldots \ldots$ I5 0

" Japonicum, Variegated. I to I $1 / 2 \mathrm{ft} . \ldots \ldots \ldots 4$ oo

2400

I 200

I 800

3300

Nandina domestica. Red berries. I to I $1 / 2 \mathrm{ft} . \ldots \ldots \ldots \ldots$ i 8 oo

" domestica. White berries. I to I $1 / 2 \mathrm{ft} . \ldots \ldots \ldots$

Olea aquifolium. I to $\mathrm{I} / 2 \mathrm{ft} \ldots \ldots \ldots \ldots \ldots \ldots \ldots \ldots \ldots$

" aquifolium, Variegated. I to I $\mathrm{I} / 2 \mathrm{ft} \ldots \ldots \ldots \ldots \ldots 275$

" fragrans. Golden yellow flowers. I to I $1 / 2 \mathrm{ft} \ldots \ldots \ldots 225$

" fragrans. White flowers. I to I $1 / 2 \mathrm{ft} \ldots \ldots \ldots \ldots 225$

" fragrans. Everlasting bloomer. I to I $\mathrm{I} / 2 \mathrm{ft} \ldots \ldots \ldots 225$

Photinia glabra. I to $\mathrm{I} / 2 \mathrm{ft} \ldots \ldots \ldots \ldots \ldots \ldots \ldots \ldots$ I 50

Pittosporum Tobira. I to $\mathrm{I} / 2 \mathrm{ft} . \ldots \ldots \ldots \ldots \ldots \ldots$

"Tobira, Yellow and White Variegated. I to

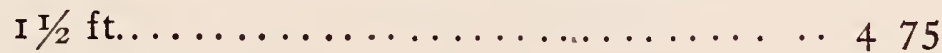

Quercus acuta. I to I $\mathrm{I} / 2 \mathrm{ft} \ldots \ldots \ldots \ldots \ldots \ldots \ldots \ldots \ldots$ I 50

" crispula (deciduous). I to $\mathrm{I} / 2 \mathrm{ft} \ldots \ldots \ldots \ldots$ I 50 
Quercus cuspidata. I to $\mathrm{I} / \mathrm{z} \mathrm{ft} . \ldots \ldots \ldots \ldots \ldots \ldots \ldots \ldots \ldots$

“ dentata (deciduous). I to I $1 / 2 \mathrm{ft} \ldots \ldots \ldots \ldots \ldots$ I 50

“ dentata aurea. I to I $1 / 2 \mathrm{ft} \ldots \ldots \ldots \ldots \ldots \ldots \ldots \ldots \ldots \ldots$

"glandulifera (deciduous). I to I $1 / 2 \mathrm{ft} \ldots \ldots \ldots \ldots 25$

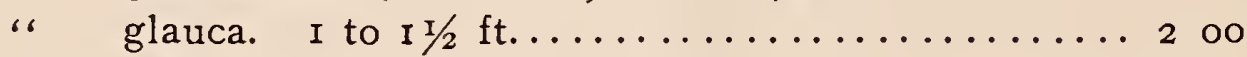

" lacera. I to $I I / 2 \mathrm{ft} \ldots \ldots \ldots \ldots \ldots \ldots \ldots \ldots \ldots$

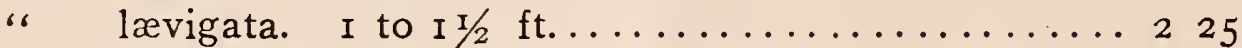

“ phyllireoides. I to I $\mathrm{I} / 2 \mathrm{ft} . \ldots \ldots \ldots \ldots \ldots \ldots$ oo

" pinnatifida (deciduous). I to I $1 / 2 \mathrm{ft} \ldots \ldots \ldots \ldots 300$

“ serrata (deciduous). I to $\mathrm{I} / 2 \mathrm{ft} \ldots \ldots \ldots \ldots \ldots 2$ oo

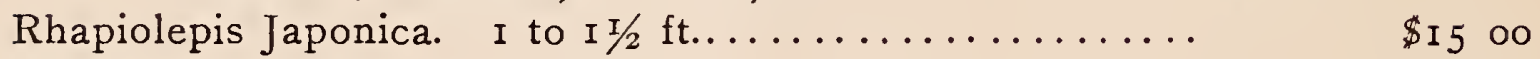

Skimmia Japonica. 8 to ro in.................. 3300

“Japonica, Variegated............... 550

Ternstrœmia Japonica. I to $\mathrm{I} / 2 \mathrm{ft} \ldots \ldots \ldots \ldots \ldots \ldots \ldots$ I 50

" Japonica, Reddish Variegated. I to I $1 / 2 \mathrm{ft} . \ldots 400$

“ Japonica, White Variegated.......... 575

Viburnum Japonicum.............................

" odoratissimum ................. 2 oо

" odoratissimum, Variegated .......... 475

\section{Conifers}

Abies bicolor (pot-grown). 8 to $\mathrm{I} 2$ in............... 375

“ brachyphylla (pot-grown). I to $\mathrm{I} / 2 \mathrm{ft} \ldots \ldots \ldots \ldots 375$

“firma (pot-grown). I to $2 \mathrm{ft} \ldots \ldots \ldots \ldots \ldots \ldots \ldots 275$

“Tomoni (pot-grown). I to I $1 / 2 \mathrm{ft} \ldots \ldots \ldots \ldots \ldots \ldots 60$

“Toramoni (pot-grown). I to I $1 / 2 \mathrm{ft} \ldots \ldots \ldots \ldots \ldots \ldots 375$

"Veitchii (pot-grown). I to $2 \mathrm{ft} \ldots \ldots \ldots \ldots \ldots \ldots \ldots \ldots$

Cephalotaxus drupacea. I to $2 \mathrm{ft} \ldots \ldots \ldots \ldots \ldots \ldots \ldots \ldots \ldots$

Cryptomeria Japonica (pot-grown). I to $2 \mathrm{ft} \ldots \ldots \ldots \ldots \ldots$ I9 oo

"Japonica (open ground). I to $2 \mathrm{ft} . \ldots \ldots \ldots . . .650$

“ Garden Varieties-

Iindai-sugi. 8 to 12 in........... 275

Bandai-sugi. 5 to 8 in................ 50

Yenko-sugi. 8 to 2 in................. 450

Ko-yo-sugi. I to I $1 / 2 \mathrm{ft} \ldots \ldots \ldots \ldots \ldots \ldots 250$

Aya-sugi. 8 to 2 in...................... 40

Howo-sugi. 8 to 12 in........... 450

Sekka-sugi. 5 to 8 in............. 450

Kusari-sugi. $\quad 5$ to 8 in........... 450

Cunninghamia Sinensis. I to I $1 / 2 \mathrm{ft} \ldots \ldots \ldots \ldots \ldots \ldots \ldots \ldots \ldots$

Ginkgo biloba (Salisburia adiantifolia) ............. I 5 oo

" biloba, Variegated.................. 4 o 
Juniperus Chinensis procumbens. I to $\mathrm{I} / 2 \mathrm{ft} \ldots \ldots \ldots \ldots \$ 2 \quad$ Per io

Per 100

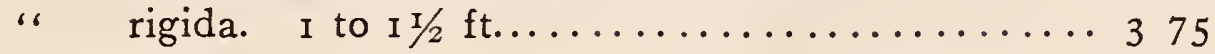

Larix leptolepis....................... 2 o

Picea Alcoquiana. I to $\mathrm{I} / 2 \mathrm{ft} \ldots \ldots \ldots \ldots \ldots \ldots \ldots \ldots \ldots \ldots \ldots \ldots$

" polita. 8 to 12 in.................... $55^{\circ}$

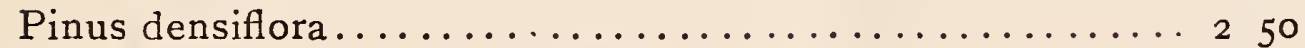

" densiflora, White Variegated............. 5 50

" densiflora, Yellow Variegated.............. 550

" Koraiensis. I ft................... 4 \%

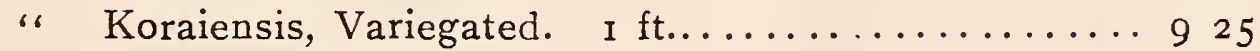

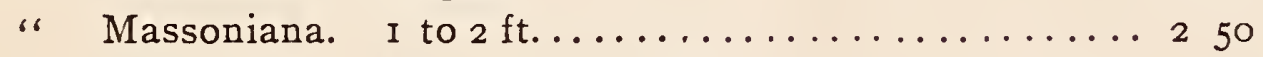

“ Massoniana, Garden Varieties-

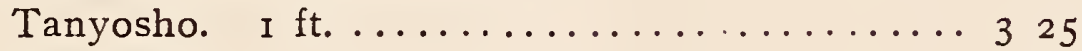

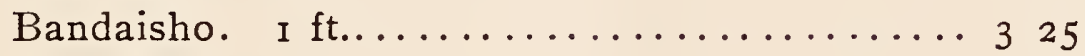

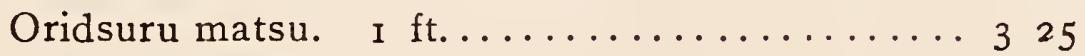

Weeping. I ft......................... 50

Sciadopitys verticillata. Io to I 2 in.............. $\$ 55$ oo

" verticillata. I to $2 \mathrm{ft} . \ldots \ldots \ldots \ldots \ldots \ldots \ldots . \ldots \ldots$

" verticillata. 2 to $3 \mathrm{ft} . \ldots \ldots \ldots \ldots \ldots \ldots \ldots$ I 10 oo

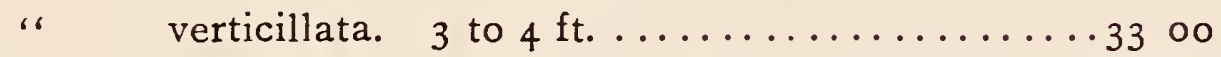

" verticillata. 4 to $6 \mathrm{ft} . \ldots \ldots \ldots \ldots \ldots \ldots \ldots \ldots$ o

" verticillata, Variegated. I ft........... 2 oo

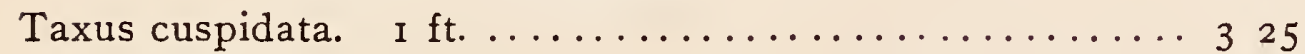

" pendula. I ft.................... 250

“ pendula, Golden Variegated. I I $2 \mathrm{ft} \ldots \ldots \ldots \ldots 250$

“ pendula, White Variegated. I $1 / 2 \mathrm{ft} \ldots \ldots \ldots \ldots \ldots 250$

" tardiva. I ft..................... 50

“ tardiva, Yellow, Variegated. I ft.......... 350

Thuja dolobrata, Variegated. I ft............. 275

" obtusa (Hinoki). I ft................ I 75

“ obtusa, Variegated, Kamakura-hiba. I ft......... I 75

“ obtusa, Variegated, Kujaku-hiba. I ft. ....... 450

" obtusa, Variegated, Hotaru-hiba. I ft........ 350

" obtusa, Variegated, Embi-hiba. I ft......... 350

“ obtusa, Variegated, Kana-ami. I to $2 \mathrm{ft} . \ldots \ldots \ldots 275$

Thuja obtusa, Famous dwarf plants, over Ioo years old-

“ obtusa, var. Chabo-hiba....per plant, $\$ 7.50$ to $\$ 50 \ldots$

" obtusa, var. Chabo-hiba, Silver Variegated ........

Per plant, \$10 to \$100..

“ obtusa, var. Chabo-hiba, Golden Variegated........

Per plant, $\$ 7.50$ to $\$$ ioo.

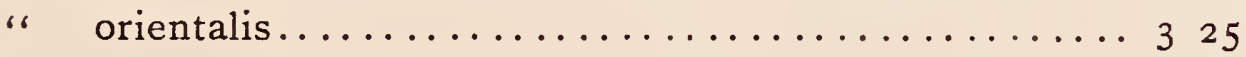

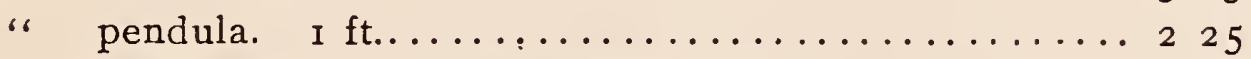

“ pendula, Golden Variegated. I I $1 / 2 \mathrm{ft} \ldots \ldots \ldots \ldots \ldots 225$

“ pendula, White Variegated. I I $/ 2 \mathrm{ft} \ldots \ldots \ldots \ldots \ldots 25$ 


\section{Hedge and Street Plants and Trees}

Acanthopanax aculeatum...................................... $\$ 2650$

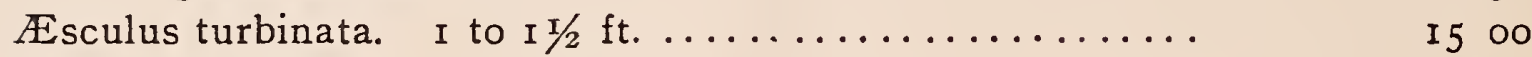

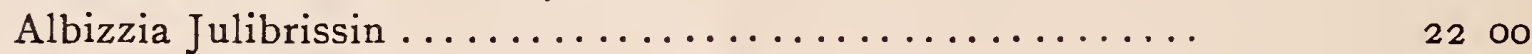

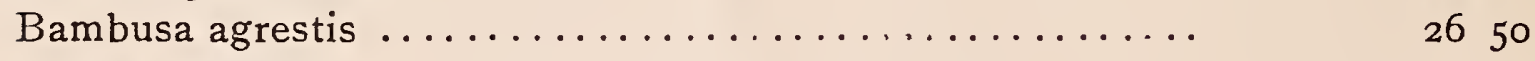

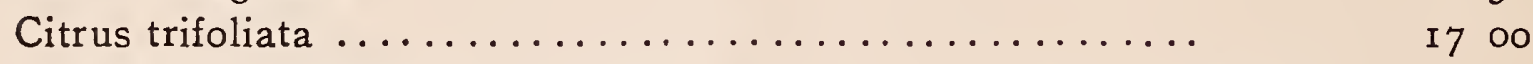

Cladrastis Amurensis. I to $2 \mathrm{ft} . \ldots \ldots \ldots \ldots \ldots \ldots \ldots \ldots \ldots$

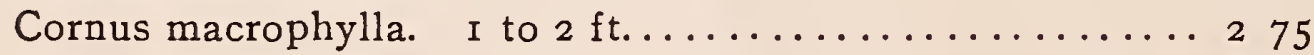

Distylium racemosum $\ldots \ldots \ldots \ldots \ldots \ldots \ldots \ldots \ldots \ldots \ldots$

Euonymus Japonica .................... I 75

Hibiscus Syriacus $\ldots \ldots \ldots \ldots \ldots \ldots \ldots \ldots \ldots \ldots \ldots \ldots \ldots$

Ilex crenata (Ornamental Evergreen) ............ 4 o

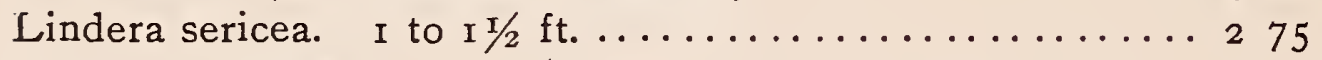

Magnolia hypoleuca. I to $2 \mathrm{ft} \ldots \ldots \ldots \ldots \ldots \ldots \ldots$ I 75

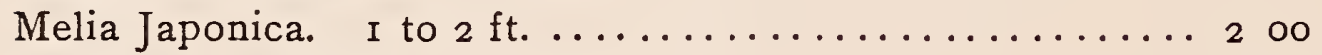

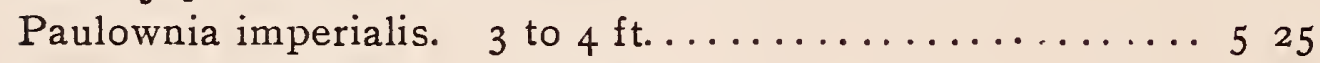

Sterculia platanifolia. I to $\mathrm{I} / 2 \mathrm{ft} \ldots \ldots \ldots \ldots \ldots \ldots \ldots \ldots$ I 50

I9 00

I I 00

3300

I 500

4675

“ platanifolia, Variegated. I to $\mathrm{I} / 2 \mathrm{ft} \ldots \ldots \ldots \ldots \ldots 475$

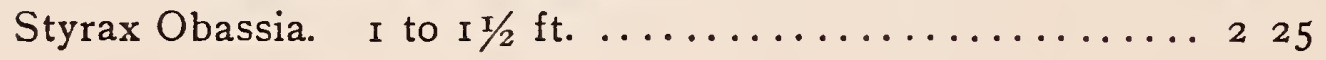

I9 00

\section{Palms, Cycas, Bamboos, Grasses and other Ornamental Plants}

Per 10 Per roo

Arundinaria Hindsii. I to $\mathrm{I} / 2 \mathrm{ft} . \ldots \ldots \ldots \ldots \ldots \ldots \ldots \ldots \ldots$ \$I9 00

" Hindsii graminea. I $1 / 2$ to $2 \mathrm{~T} / 2 \mathrm{ft} \ldots \ldots \ldots \ldots \ldots$ I9 00

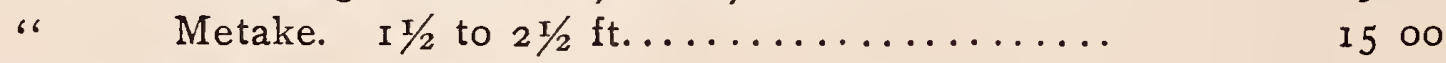

"Narihira. I $1 / 2$ to $2 \mathrm{ft} . \ldots \ldots \ldots \ldots \ldots \ldots \ldots$ I9 00

Aspidistra lurida, Green..........per r, 000 leaves, $\$ 37 \ldots$

" " Variegated ..... " " " 6 " $\$ 66 \ldots$

Bambusa agrestis. I to $\mathrm{I} / 2 \mathrm{ft} . \ldots \ldots \ldots \ldots \ldots \ldots \ldots \ldots$

" agrestis, Variegated. I $1 / 2$ to $2 \mathrm{ft} . \ldots \ldots \ldots \ldots \ldots$

" argento striata. I $\mathrm{x} / 2$ to $2 \mathrm{ft} \ldots \ldots \ldots \ldots \ldots \ldots \ldots$

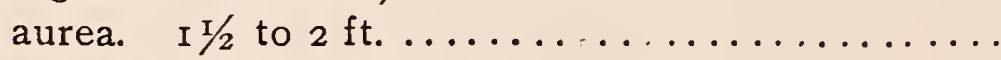

aurea striata. I $\mathrm{I} / 2$ to $2 \mathrm{ft} . \ldots \ldots \ldots \ldots \ldots \ldots \ldots$

aurea. Leaves and stems variegated. I to $2 \mathrm{ft}$...

Henonis (Hachiku). I to $2 \mathrm{I} / 2 \mathrm{ft} \ldots \ldots \ldots \ldots \ldots$

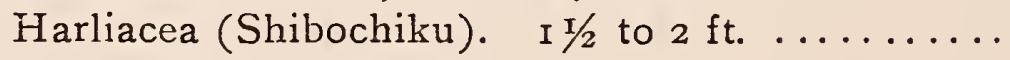

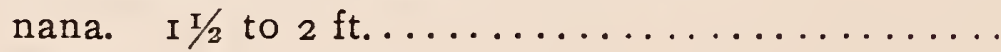

2200

2650

I9 00

I6 oo

I9 00

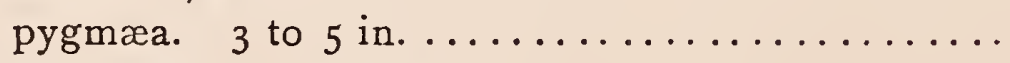

I 8 oo

2200

Suo-chiku. I $1 / 2$ to $2 \mathrm{ft} . \ldots \ldots \ldots \ldots \ldots \ldots \ldots$

2200

"Taiho-chiku. I $1 / 2$ to $2 \mathrm{ft} . \ldots \ldots \ldots \ldots \ldots \ldots$ 
Cyathea spinulosa, Tree Fern. Of easy cultivation ; requires

the same treatment as Cycas revoluta. Sizes, 6 in. to $\mathrm{I} / 2 \mathrm{ft}$.

Cycas revoluta stems. $1 / 2$ to I $\mathrm{ft} . .$. . per $\mathrm{I}, 000 \mathrm{lbs} ., \$ 82 \ldots$

" " " I to $3 \mathrm{ft} \ldots \ldots$ ". " $\$ 77 \ldots$

Carex Gaudichaudiana...................\$4 75

Eulalia Japonica (clumps)

3300

I9 00

"Japonica zebrina (clumps) ...............

" natsubo (clumps) ...................

" sempervirens (clumps) $\ldots \ldots \ldots \ldots \ldots \ldots \ldots \ldots \ldots$

Miscanthus sacchariflorus (Ogi) (clumps) $\ldots \ldots \ldots \ldots \ldots$

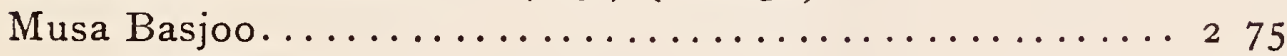

" coccinea ..................... 275

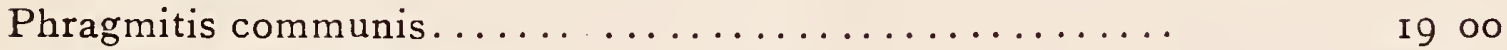

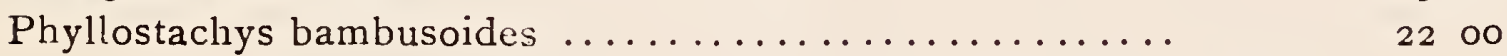

" heterocycla .................. 475

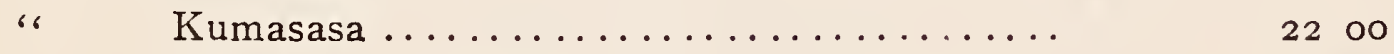

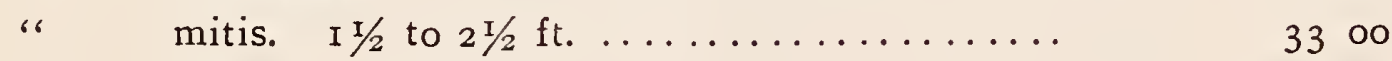

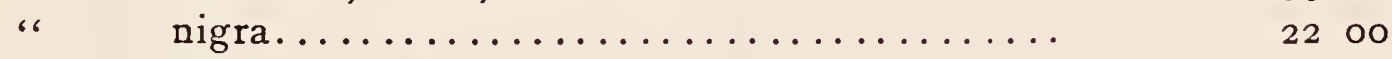

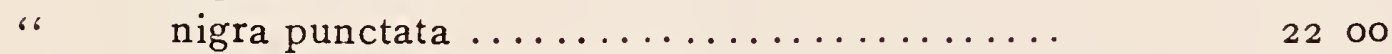

"quadrangularis. I $\mathrm{t} / 2$ to $2 \mathrm{ft} \ldots \ldots \ldots \ldots \ldots \ldots$.

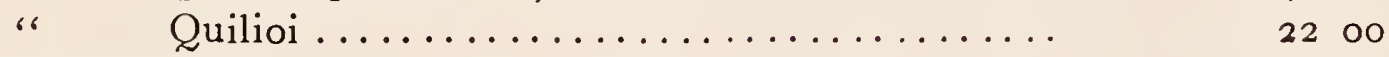

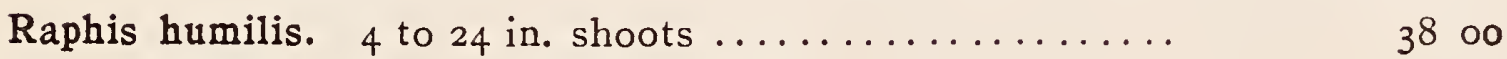

"flabelliformis. 4 to $I 8$ in. shoots ........... 3300

\section{Japanese Fruit Trees and Plants}

Actinidia arguta. I to $\mathrm{I} / 2 \mathrm{ft} . \ldots \ldots \ldots \ldots \ldots \ldots \ldots \ldots \ldots$

Castanea vulgaris Tamba. Mammoth Chestnut. Open

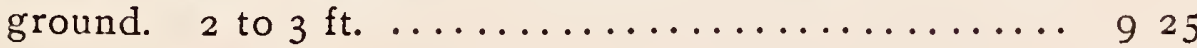

Citrus, or Orange Trees-

"Aurantium. I to $I / 2 \mathrm{ft} . \ldots \ldots \ldots \ldots \ldots \ldots \ldots$

“ Bigaradia (Bitter Orange). I to $I / 2 \mathrm{ft} . \ldots \ldots \ldots$

" Decumana. I $1 / 2$ to $2 \mathrm{ft} . \ldots \ldots \ldots \ldots \ldots \ldots \ldots$

" Japonica. Variety with elliptical fruit. I to I $1 / 2 \mathrm{ft} . .2400$

" Japonica. Variety with round fruit. I to I I $/ 2 \mathrm{ft} . \ldots 2400$

" Japonica Omi-Kinkan. Large, round ......... 2700

" media chirocarpus.................. 27 o०

" nobilis, var. Kino-Kuni. I to I $1 / 2 \mathrm{ft} \ldots \ldots \ldots \ldots . \ldots 2400$

" nobilis, var. Unshin ................ 24 oo

" nobilis, var. Satsuma ............... 30 ००

" trifoliata. Best for grafting. I to $\mathrm{I} / 2 \mathrm{ft} . \ldots \ldots$ I7 00

“ Summer Variety. I to I $1 / 2 \mathrm{ft}$., pot-grown ....... 
Diospyros Kaki, Japanese Persimmons. Varieties which

Hyakume. Oblong, very large. 3 to $4 \mathrm{ft} \ldots \ldots \ldots \ldots$ II oo Zenji. Medium. 3 to $4 \mathrm{ft} . \ldots \ldots \ldots \ldots \ldots$ I I oo

$\$$ IOO OO

Kuro-Kuma. Flattened ................ I I oo

IOO 00

Nitari. Pointed. 3 to $4 \mathrm{ft} . \ldots \ldots \ldots \ldots \ldots \ldots$ I I oo

IOO 00

Gosho-gaki. 3 to $4 \mathrm{ft} . \ldots \ldots \ldots \ldots \ldots \ldots \ldots$ I I oo

100 OO

Daidai-maru. 3 to $4 \mathrm{ft} . \ldots \ldots \ldots \ldots \ldots \ldots$ I I oo

IOO 00

Tsuru-no-Ko. 3 to $4 \mathrm{ft} . \ldots \ldots \ldots \ldots \ldots \ldots$ I I oo IOO Ioo oo

Varieties which lose their stringency only when subjected to so-called improvement-

Hachiya. 3 to $4 \mathrm{ft} \ldots \ldots \ldots \ldots \ldots \ldots \ldots \ldots$ I I oo

Yemon. 3 to $4 \mathrm{ft} . \ldots \ldots \ldots \ldots \ldots \ldots \ldots \ldots$ I I oo

IOO 00

Yama-Yemon. 3 to $4 \mathrm{ft} \ldots \ldots \ldots \ldots \ldots \ldots$ I I 0

IOO 00

Masu-gaki. 3 to $4 \mathrm{ft} . \ldots \ldots \ldots \ldots \ldots \ldots \ldots$ I I oo

100 OO

Mino-dsuro. 3 to $4 \mathrm{ft} . \ldots \ldots \ldots \ldots \ldots \ldots \ldots$ I I oo

IOO 00

IOO 00

Elæagnus longipes. I to $\mathrm{I} / 2 \mathrm{ft} \ldots \ldots \ldots \ldots \ldots \ldots$ I3 3 oo

I IO 00

" pungens. I to $\mathrm{I} / 2 \mathrm{ft} \ldots \ldots \ldots \ldots \ldots \ldots \ldots \ldots$ I 7 oo

" umbellata. I to I $I / 2 \mathrm{ft} \ldots \ldots \ldots \ldots \ldots \ldots$ I 3 oo

I IO 00

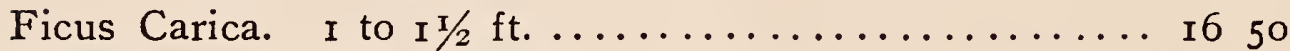

Juglans cordiformis (Walnut). 3 to $4 \mathrm{ft} \ldots \ldots \ldots \ldots$ I I oo

" regia. 3 to $4 \mathrm{ft} . \ldots \ldots \ldots \ldots \ldots \ldots \ldots \ldots$ I I oo

" Sieboldiana. 3 to $4 \mathrm{ft} \ldots \ldots \ldots \ldots \ldots \ldots \ldots$ I I oo

IOO 00

IOO.0O

IOO 00

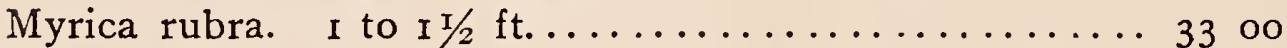

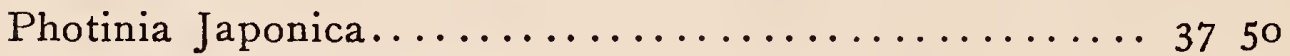

Prunus armeniaca (Apricot). I to $\mathrm{I} / 2 \mathrm{ft} \ldots \ldots \ldots \ldots 2750$

" triflora, var. Shiro-sumomo. 3 to $4 \mathrm{ft} \ldots \ldots \ldots$.... ro oo

" triflora, var. Aka-sumomo. 3 to $4 \mathrm{ft} . \ldots \ldots \ldots$ ro oo

" triflora, var. Botankyo. 3 to $4 \mathrm{ft} . \ldots \ldots \ldots \ldots$ ro oo

" triflora, var. Ko-botan. 3 to $4 \mathrm{ft} . \ldots \ldots \ldots \ldots$ ro oo

“ triflora, var. Haku-botan. 3 to $4 \mathrm{ft} . \ldots \ldots \ldots \ldots$ ro oo

" triflora, var. Furugiya-beni. 3 to $4 \mathrm{ft}$........ Io oo

" triflora, var. Hatankyo. 3 to $4 \mathrm{ft} . \ldots \ldots \ldots \ldots$ ro oo

" triflora, var. Suika-mono. 3 to $4 \mathrm{ft}$......... ro oo

"triflora, var. Large Red. Medium ripening. 3 to $4 \mathrm{ft} . \ldots \ldots \ldots \ldots \ldots \ldots \ldots$ ro oo

“ triflora, var. Large Red. Late ripening. 3 to $4 \mathrm{ft}, \ldots \ldots \ldots \ldots \ldots \ldots \ldots \ldots$ ro oo

82 oo

82 oo

82 oo

82 oo

82 oo

8200

82 oo

82 oo

82 oo

8200

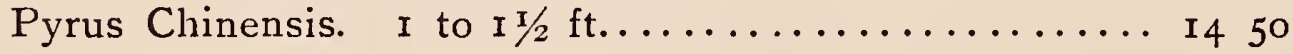

"Cydonia. I to $\mathrm{I} / 2 \mathrm{ft} . \ldots \ldots \ldots \ldots \ldots \ldots \ldots \ldots$ I 450

“Ussuriensis. Ten varieties. 3 to $4 \mathrm{ft} . \ldots \ldots \ldots . .875$

Rubus incisus (Yellow Raspberry). 2 to $4 \mathrm{ft} \ldots \ldots \ldots \ldots 450$

7100 38 oo

" sorbifolius ........................... 275

" trifidus. 2 to $3 \mathrm{ft} . \ldots \ldots \ldots \ldots \ldots \ldots \ldots \ldots$

I9 00

Vitis vinifera (Japanese Grape). 3 to $4 \mathrm{ft} . \ldots \ldots \ldots \ldots$ 


\section{Seedlings and Stock Plants}

Per 1,000

Citrus trifoliata. Pencil thickness...................\$28 ००

Cherry cuttings (for grafting plums and apples)............. 23 o०

Chestnut. Pencil thickness......................... 3300

Magnolia Kobus. Pencil thickness.................... 40 o

Maples. Pencil thickness......................... 27 o

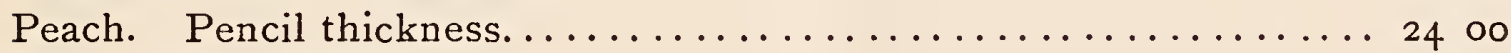

Pear. Pencil thickness......................... 22 oo

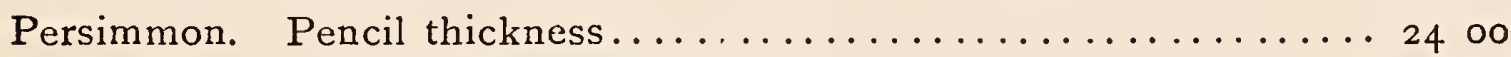

Prunus triflora. Pencil thickness ................... 2400

Pyrus Toringo. Pencil thickness.................... 2400

\section{Economic Plants}

Alnus firma. Seeds used for dyeing purposes. $2 \mathrm{ft} \ldots \ldots$ Per 100 Per $r, c o 0$

“ incana. Bark used for dyeing purposes. $2 \mathrm{ft}$..... I9 oo

Broussonetia papyrifera. For manufacturing paper. $2 \mathrm{ft} .950$ " Kajinoki. $2 \mathrm{ft} . \ldots \ldots \ldots \ldots \ldots \ldots \ldots \ldots . \ldots 90$

Camellia theifera (Tea Plant). White flowers. I to $2 \mathrm{ft} . .2200$

" theifera. Rose flower. 8 to $\mathrm{I} 2$ in............

" theifera, var. macrophylla. I ft...........

" theifera, var. macrophylla, Variegated. I ft....

Cinnamomum Camphora (Camphor Tree). I to I $1 / 2 \mathrm{ft} \ldots$ I 650

$\$ 13200$

Daphne Genkwa. For manufacturing paper. I ft...... I7 oo

Edgeworthia papyrifera. For manufacturing paper...... 750

Ipomœa Batatas (Japanese Sweet Potato). Tubers..... 2 oo

Juncus communis. For manufacturing mats. In pots....

Lindera sericea. I to $\mathrm{I} / 2 \mathrm{ft} . \ldots \ldots \ldots \ldots \ldots \ldots \ldots \ldots$

Morus alba (Mulberry). I to $\mathrm{I} / 2 \mathrm{ft} . \ldots \ldots \ldots \ldots \ldots \ldots$

Polygonum Sachalinense. Forage plant. Roots........

Pueraria Thunbergiana. For starch. Roots..........\$7 $\$ 50$

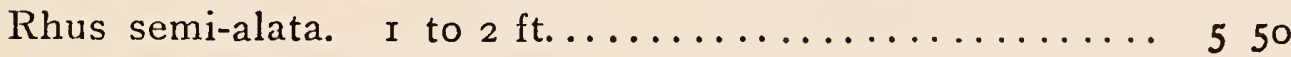

" succedanea (Wax Tree). I to $2 \mathrm{ft} \ldots \ldots \ldots \ldots \ldots \ldots 70$

" vernicifera (Lacquer Tree). I to $2 \mathrm{ft} . \ldots \ldots \ldots \ldots \ldots$

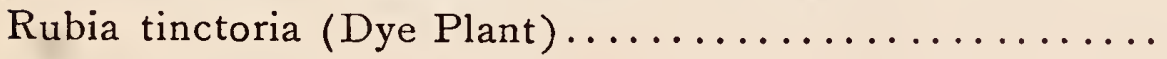

Salix multinervis. $2 \mathrm{ft} . \ldots \ldots \ldots \ldots \ldots \ldots \ldots \ldots \ldots$

Wickstrœmia pauciflora. For manufacturing Japanese copying paper..................... r7 oo

Zizania aquatica. For manufacturing Japanese mats.

Clumps........................ 8 oo 


\section{Climbers and Creepers}

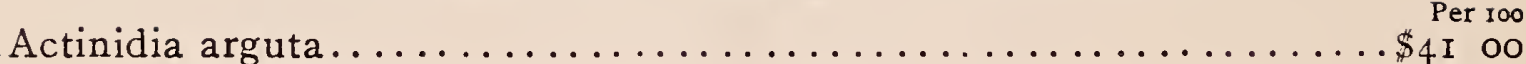

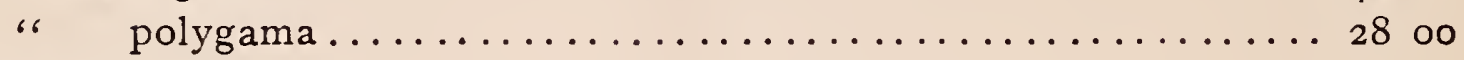

Akebia quinata..................................... 20

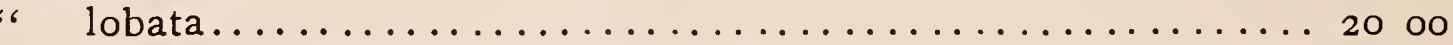

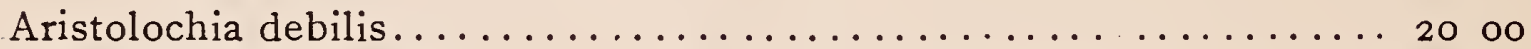

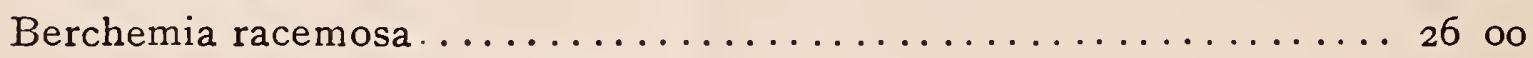

" racemosa, Variegated................... 40 oо

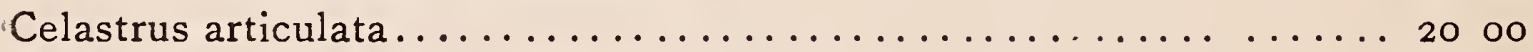

Clematis florida. Greenish white flowers................. 20 oo

" florida. Double white flowers, green-tipped.......... 20 oо

" paniculata ......................... I I 00

" patens. Large violet flowers.................. 20 oо

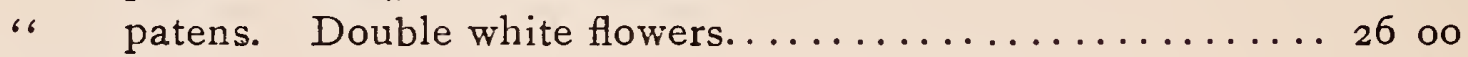

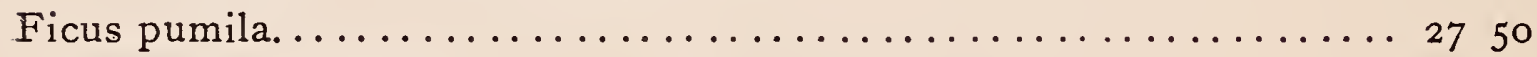

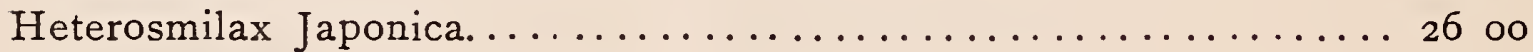

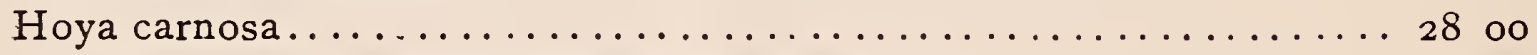

$"$ carnosa. Central variegated leaves............... 35 o

" carnosa. Marginal variegated leaves............... 35 о

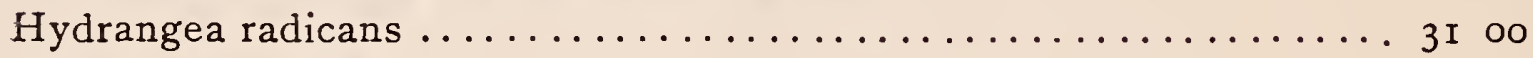

Kadsura Japonica............................ I9 oo

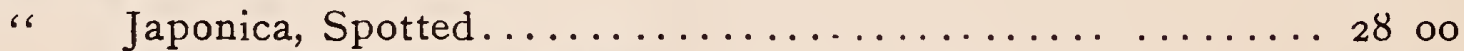

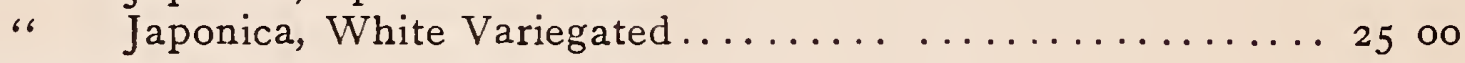

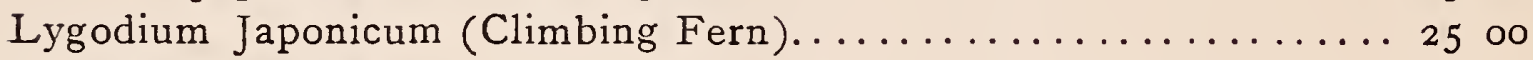

Millettia Japonica.......................... I9 oo

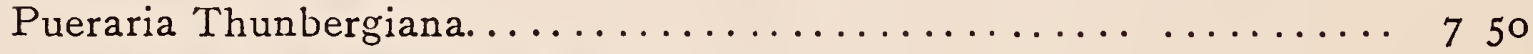

Rubus rosifolius, var. coronarius.................. I9 oo

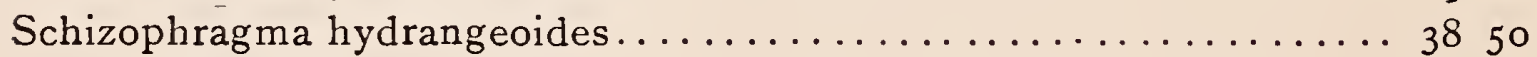

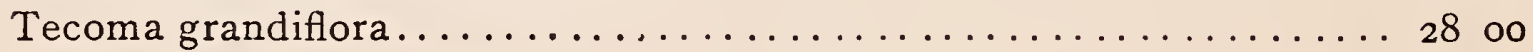

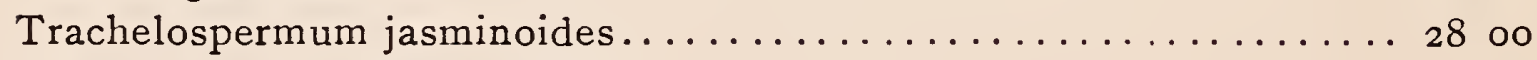

Wistaria brachybotrys, White . . ................ 33 о

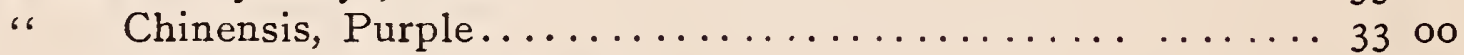

“ Chinensis, Purple, Double.................. 55 oo

\section{Aquatic Plants}

Carex Gaudichaudiana. Clumps.................. 47 о

Euriale ferox (see under Seeds).

Nelumbium speciosum. Kinds : Large Pure White, Large Light Rose,

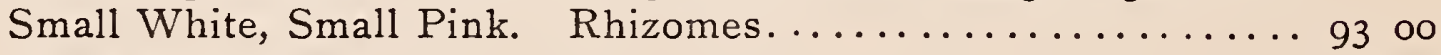

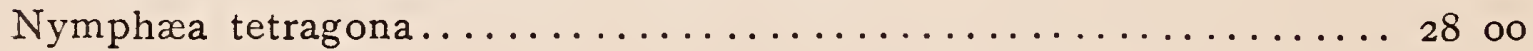

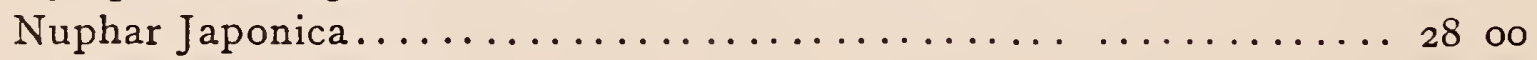

Sagittaria sagittæfolia............................. 225

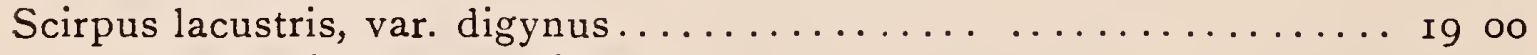

" lacustris, var. genuinus.................... I9 00

Trapa bispinosa (see under Seeds). 


\section{Hardy Perennials}

Adonis Davurica, Single ......................\$ \$ 50

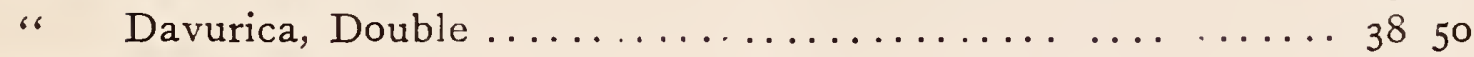

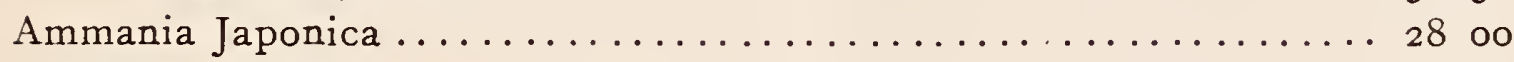

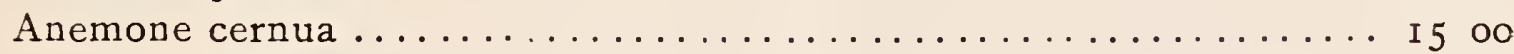

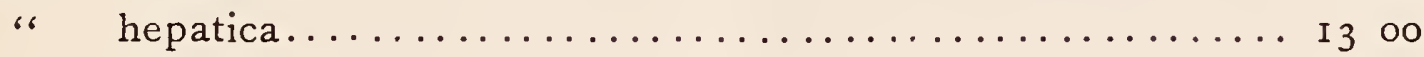

" Japonica............................ I 5 oo

Anthericum yedoense........................... I9 oo

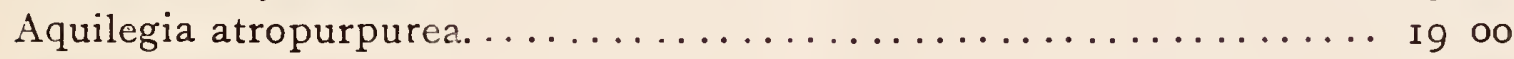

Aralia cordata............................... I 5 oo

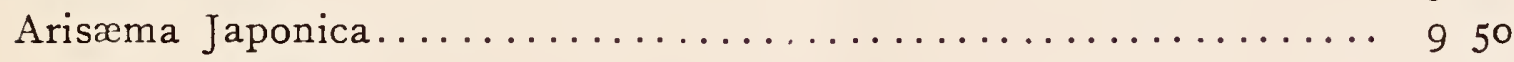

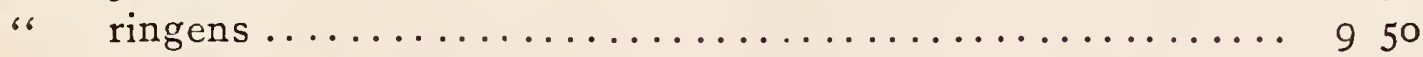

Asarum Sieboldii............................. I9 oo

"Blumei. More than twenty varieties ............... I9 oo

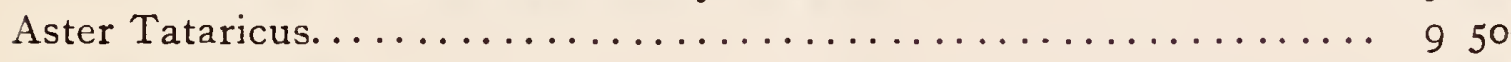

Astilbe Japonica.............................. I3 00

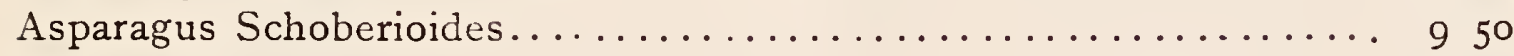

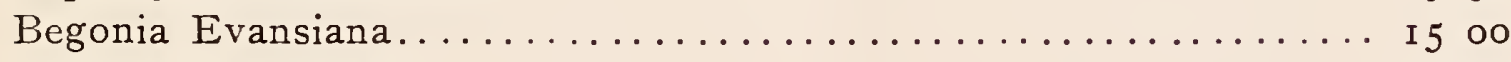

Campanula punctata..................... I I oo

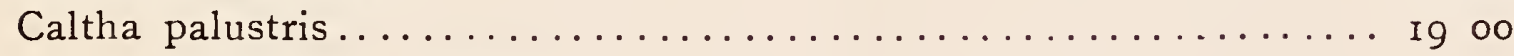

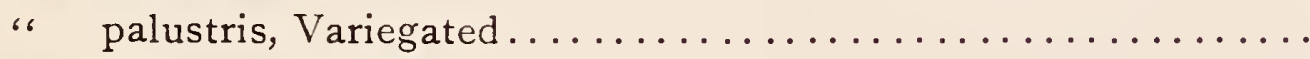

Conandron ramondioides....................... I I oo

Conophallus Konjak........................... Io oo

Epimedium macranthum....................... Io oo

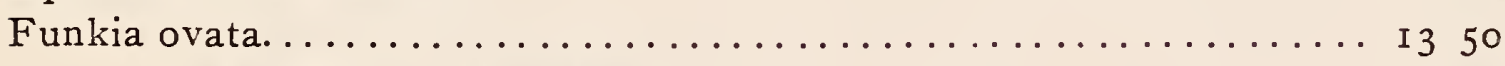

" ovata, White Variegated.................... I9 oo

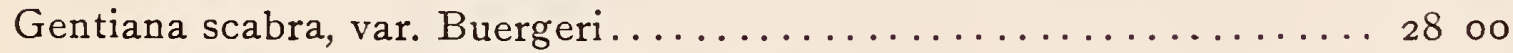

" scabra, var. Buergeri, Variegated............... 3700

Leucanthem $\operatorname{articum} \ldots \ldots \ldots \ldots \ldots \ldots \ldots \ldots \ldots \ldots \ldots$ i9 oo

" nipponicum ...................... 19 oo

Lychnis grandiflora.......................... I 2 oo

" Senno ............................. 12 oo

Senno. Mottled and striped flowers ................ I 2 oo

Millettia Japonica ........................... I9 oo

Parnassia palustris ... ..................... n

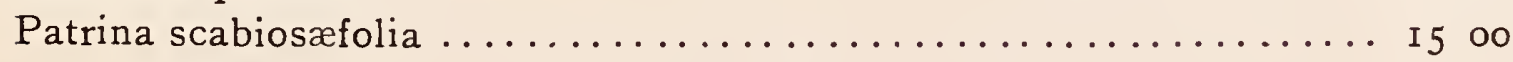

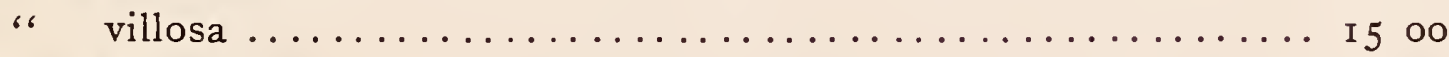

Physalis Francheti ........................... I 3 oo

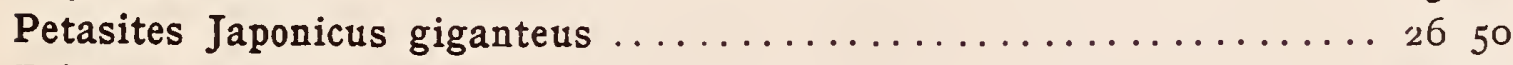

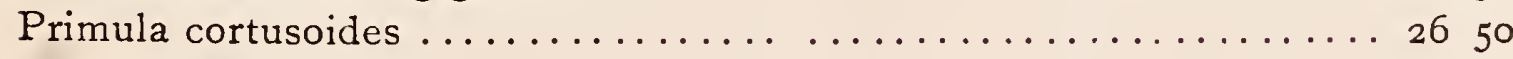

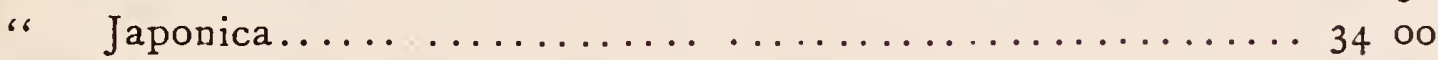

Rhodea Japonica, var. Miyako..................... 80 o

“ Japonica, var. Takakuma .................... 80 oo

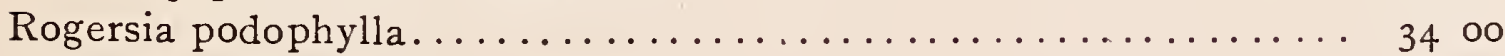

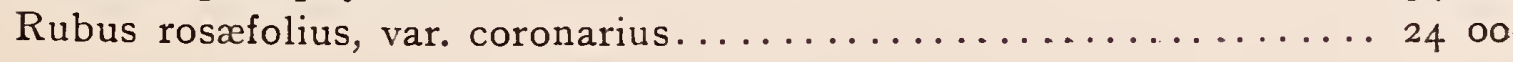




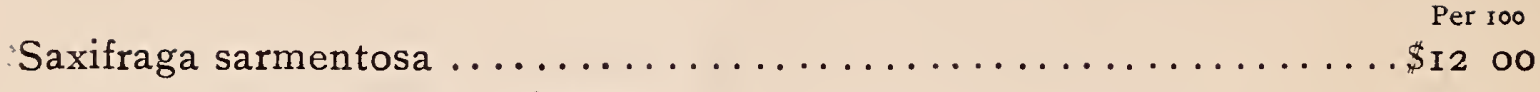

" sarmentosa, Variegated $\ldots \ldots \ldots \ldots \ldots \ldots \ldots \ldots \ldots \ldots \ldots$ I2 0 o

Schizocodon soldanelloides........................ 24 ००

Sedum Sieboldii $\ldots \ldots \ldots \ldots \ldots \ldots \ldots \ldots \ldots \ldots \ldots \ldots \ldots \ldots \ldots$ I 8 oo

" Sieboldii, Variegated $\ldots \ldots \ldots \ldots \ldots \ldots \ldots \ldots \ldots \ldots \ldots \ldots \ldots \ldots$ 18 $8 \ldots$

Senecio Kæmpferi............................ 33 o

"Kæmpferi, Variegated..................... 33 о

Simicifuga $\operatorname{simplex} \ldots \ldots \ldots \ldots \ldots \ldots \ldots \ldots \ldots \ldots \ldots \ldots$ r 8 oo

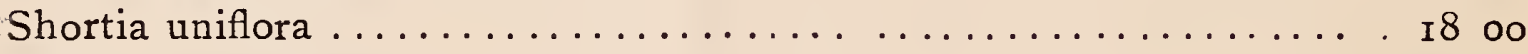

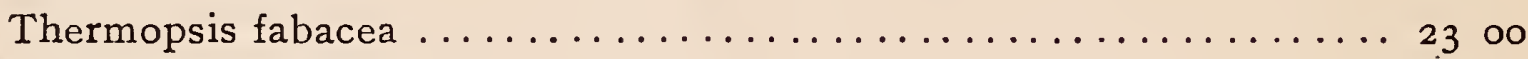

Tricyrtis Japonica.......................... I8 o

\section{Seed Specialties}

(No order accepted for less than one pound)

Japanese Imperial Morning-glory- $\quad$ Per rolbs. Per roolbs.

Best Single........................\$ \$6 $50 \quad \$ 53$ o

Best Single Fringed .................... 20 oo I60 00

Best Double ........................ 32 o

Best Yellow ...................... 66 o

Giant "Mikado" .................... 4650

Dolichos, "Daylight" (White Hyacinth Bean) ........ $725 \quad 6600$

Castanea vulgaris (Mammoth Chestnut) ........... 33 oo

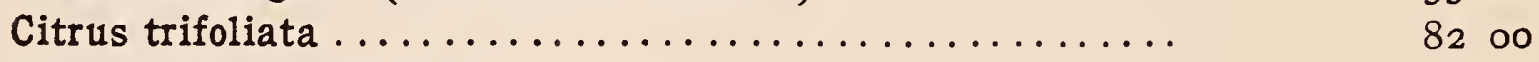

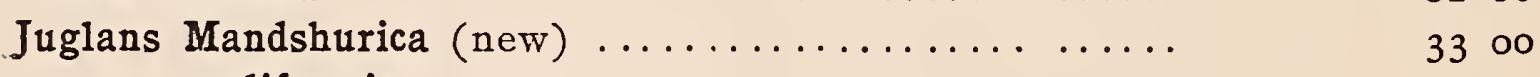

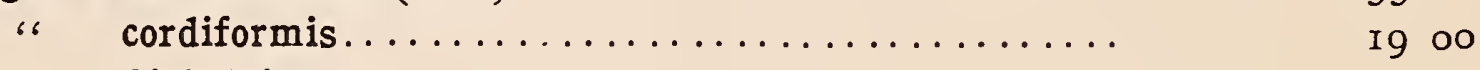

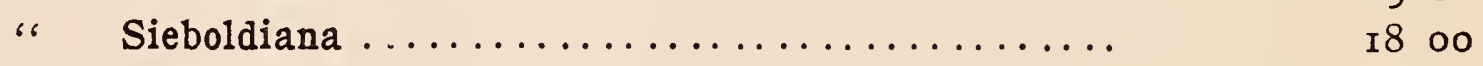

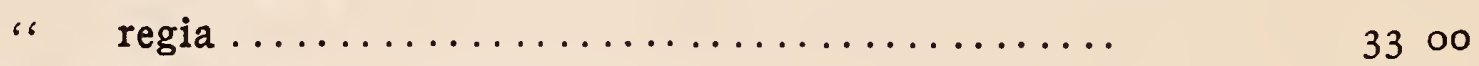

Pyrus Ussuriensis (Japanese Pear seed) $\ldots \ldots \ldots \ldots \ldots \ldots .248$ o०

\section{Tree and Shrub Seeds}

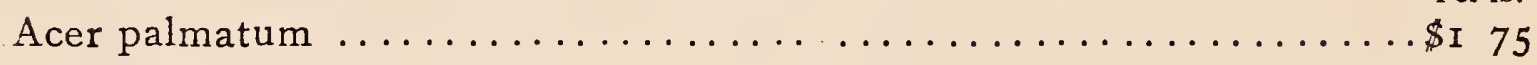

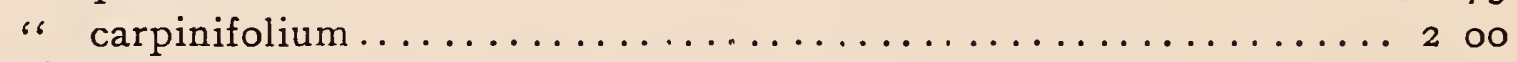

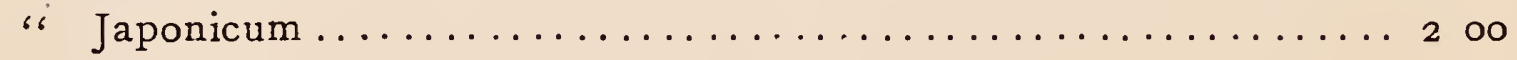

" Nikoense ............................ 2 o

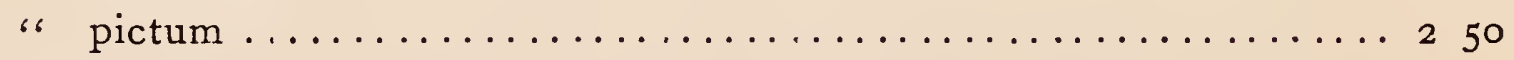

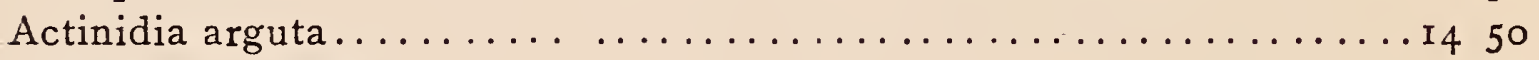

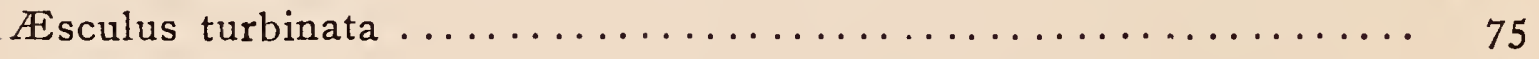

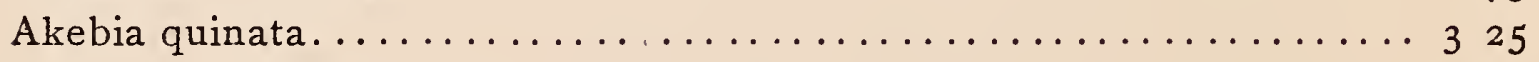

Albizzia Julibrissin . . . . . . . . . . . . . . . . . . . 250

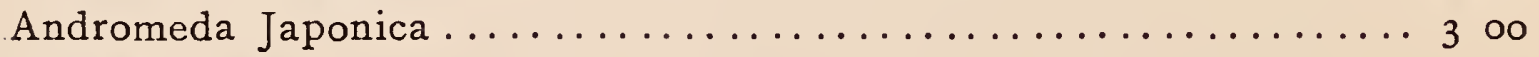

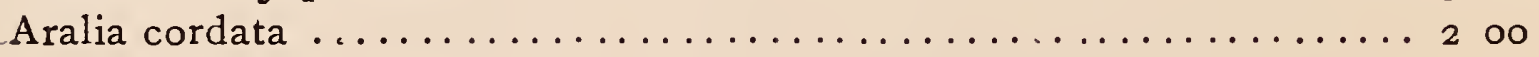

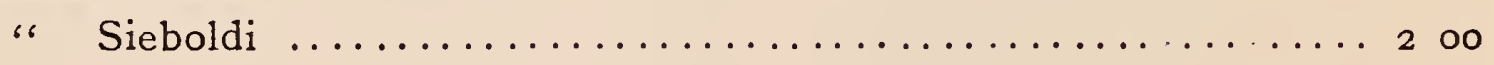


Aralia spinosa

Ardisia crispa

"Japonica

300

Berchemia racemosa.

300

Buxus Japonica

Callicarpa Japonica.

275

Camellia Japonica.

200

"Sasanqua

300

Carpinus Yedoensis.

I 00

Cedrela Chinensis . .

I 50

Celtis Chinensis.

275

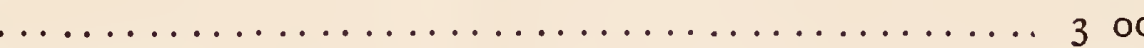

Cercidiphyllum Japonicum ......................... 3 o

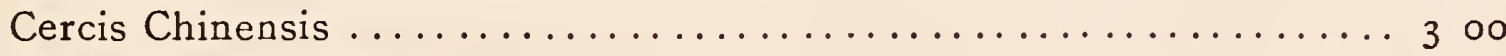

Celastrus articulatus........................... 3 \%

Cinnamomum Camphora......................... I 25

" pedunculatum $\ldots \ldots \ldots \ldots \ldots \ldots \ldots \ldots \ldots \ldots$ I 25

" Loureiri ........................ I 75

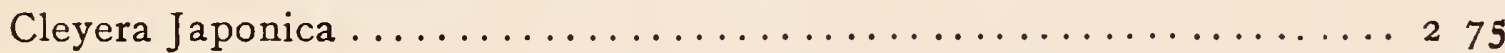

Cornus Kousa............................ I 75

" macrophylla .......................... I 75

$"$ officinalis............................. 3 oo

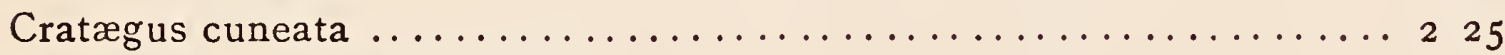

Daphniphyllum macropodum...................... I 25

Deutzia gracilis ............................... I 75

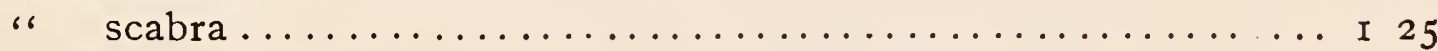

Diospyros Kaki ............................ I o

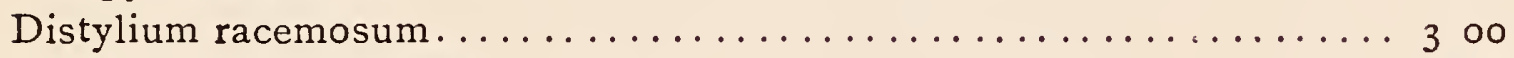

Edgeworthia papyrifera.......................... I 25

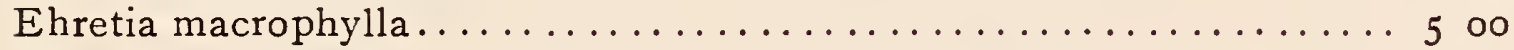

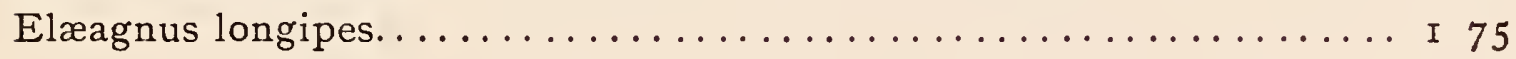

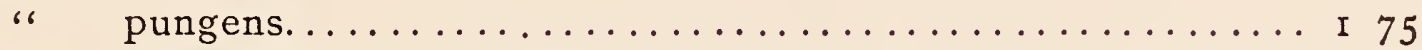

" umbellatus $\ldots \ldots \ldots \ldots \ldots \ldots \ldots \ldots \ldots \ldots \ldots \ldots \ldots \ldots \ldots$ I 75

Elæococca cordata ........................... I oo

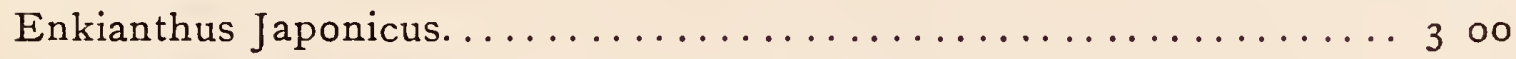

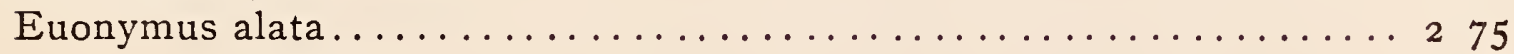

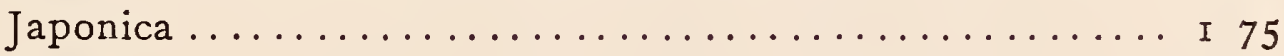

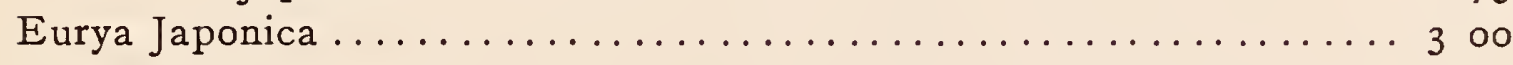

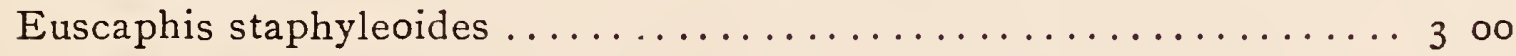

Fagus sylvatica.......................... I 75

Gardenia florida.............................. 2 o o

Gleditschia Japonica. ........................ I o

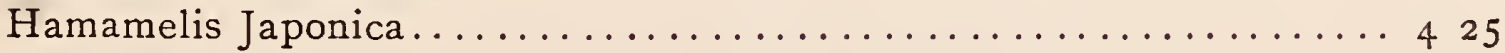

Hibiscus Hamabo........................... I 75

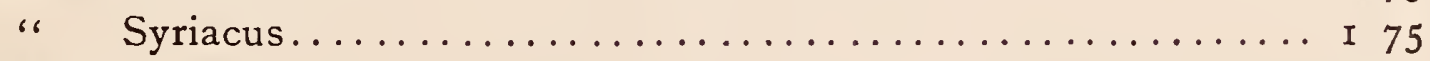

Hovenia dulcis............................. I

Idesia polycarpa............................ 675 


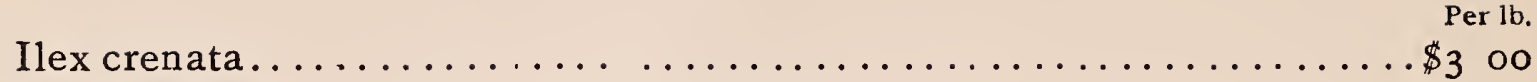

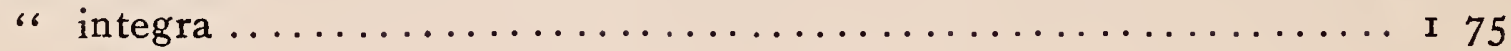

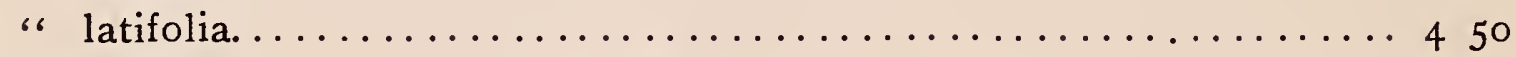

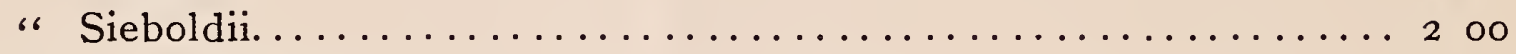

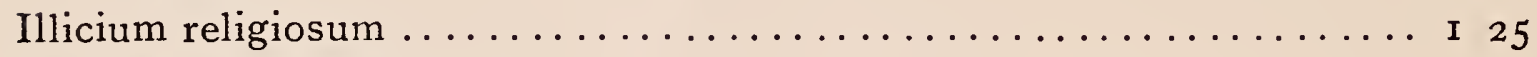

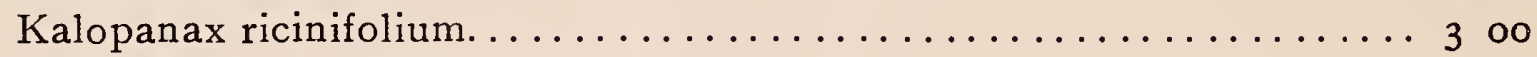

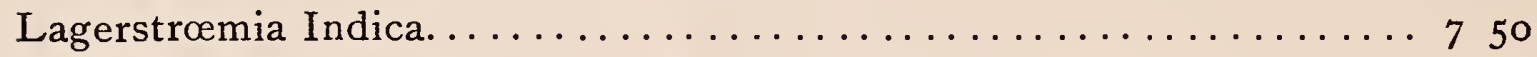

Lespedeza bicolor........................... 3 o

" bicolor, White-flowering................ 5 oо

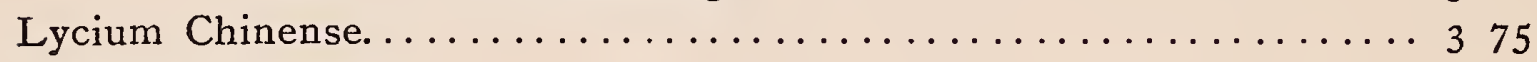

Magnolia hypoleuca $\ldots \ldots \ldots \ldots \ldots \ldots \ldots \ldots \ldots \ldots \ldots \ldots \ldots \ldots \ldots \ldots \ldots$

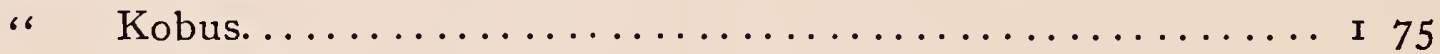

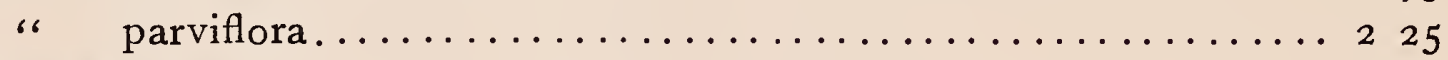

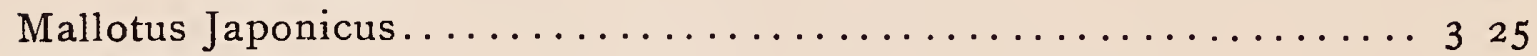

Melia Japonica........................... I

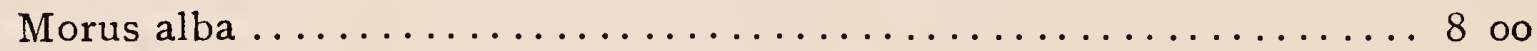

Myrica rubra............................... I 75

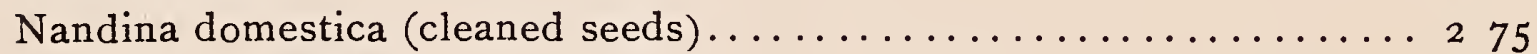

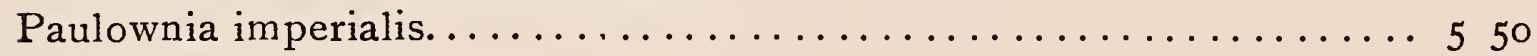

Photinia glabra................................. 2 o

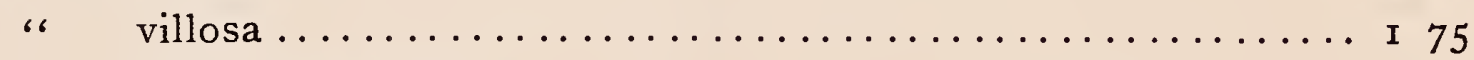

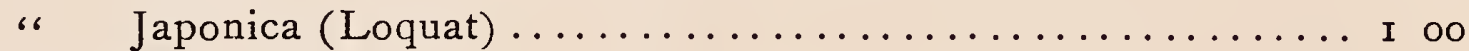

Pittosporum Tobira.......................... I 75

Prunus Japonica......................... I 75

" Mume (Japan Flowering Plum) ................ I o

“ Pseudo-Cerasus (Japan Flowering Cherry)............. I 75

" triflora............................. I

Pterocarya rhoifolia............................ 250

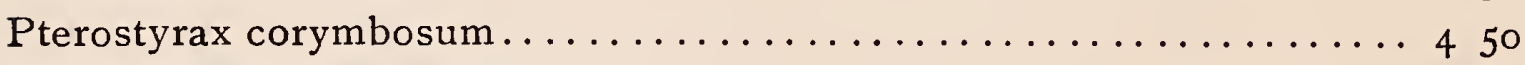

Pyrus Toringa, var. incisa..................... I 75

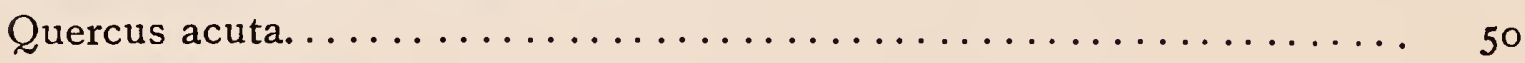

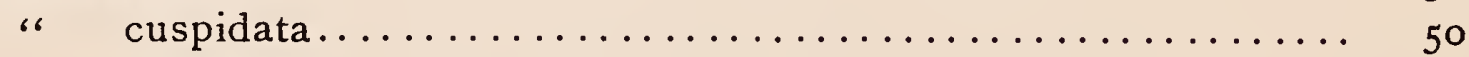

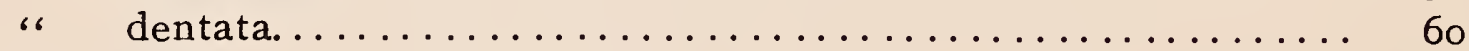

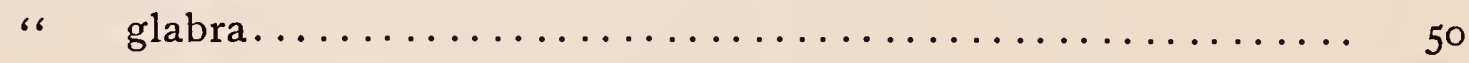

" phillyræoides $\ldots \ldots \ldots \ldots \ldots \ldots \ldots \ldots \ldots \ldots \ldots \ldots \ldots \ldots$ I

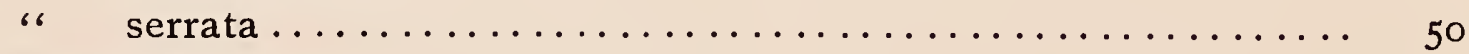

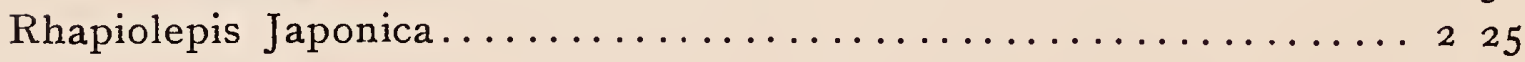

Rhus succedanea............................ I 50

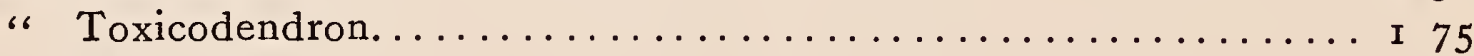

$"$ vernicifera.......................... I 50

Rhodotypos kerrioides.......................... 550

Rosa multiflora (Japanese Wild Rose)................. 2 oо

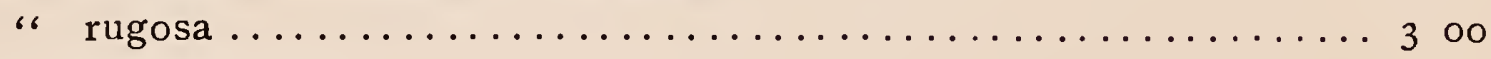

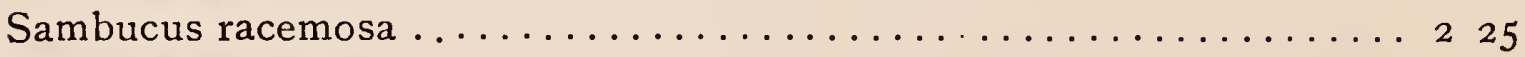

Skimmia Japonica............................. 225 
Sophora Japonica . . . . . . . . . . .

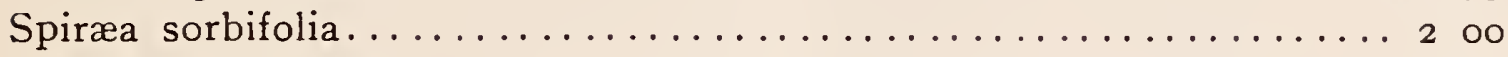

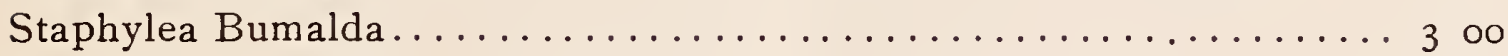

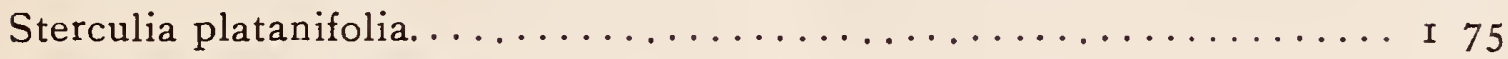

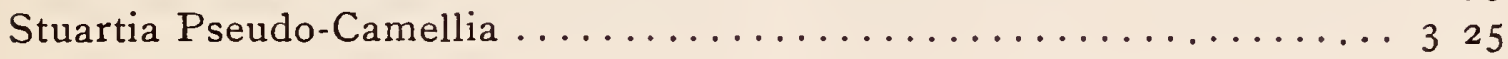

Styrax Japonica............................ I o

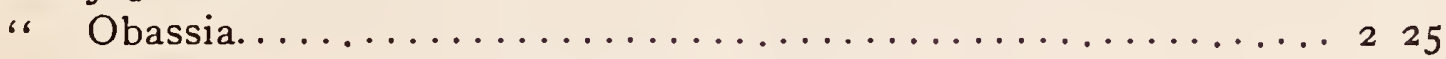

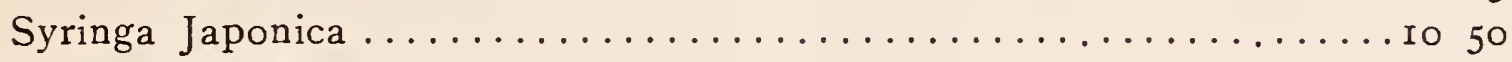

Thea viridis (Tea Plant) $\ldots \ldots \ldots \ldots \ldots \ldots \ldots \ldots \ldots \ldots \ldots \ldots \ldots$ I

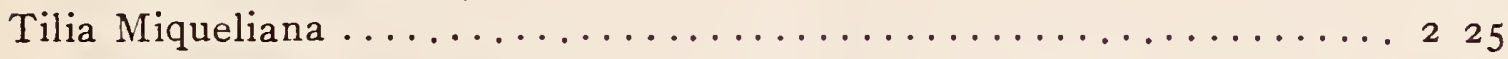

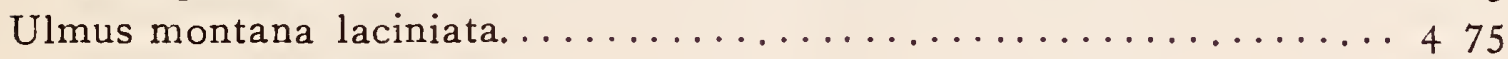

Viburnum dilatatum....................... I 75

" odoratissimum ..................... I 75

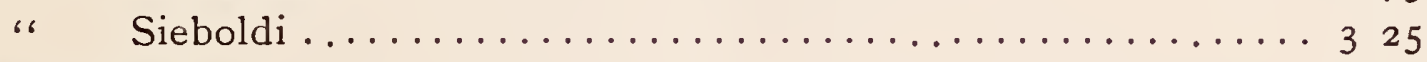

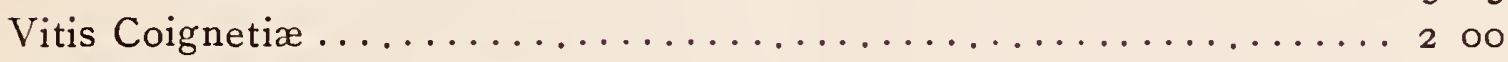

Wistaria Chinensis. Purple..................... I 75

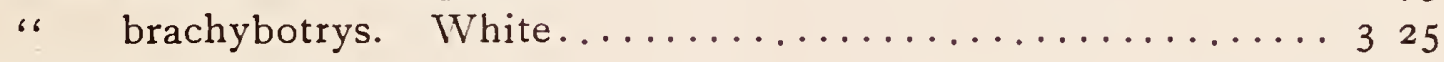

Zanthoxylum piperitum $\ldots \ldots \ldots \ldots \ldots \ldots \ldots \ldots \ldots \ldots \ldots \ldots$ r

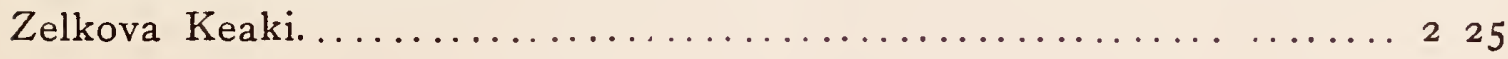

\section{Conifer Seed}

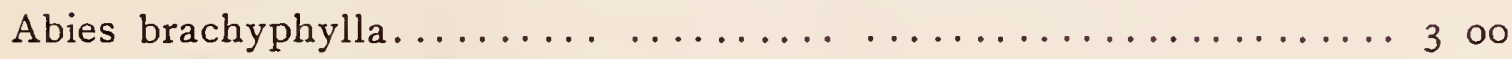

$"$ firma........................... I 75

" Mariesii ............................ 5 o

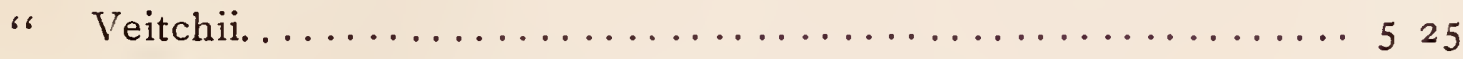

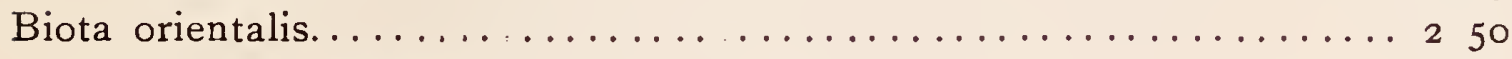

Cephalotaxus drupacea......................... r o

Chamæcyparis obtusa.......................... I 25

Cryptomeria Japonica .................................

Ginkgo biloba ........................... r o

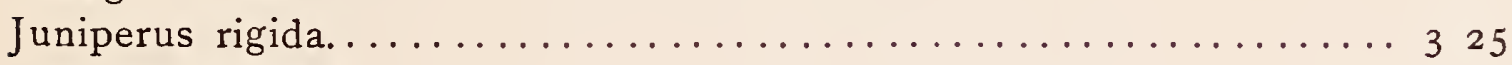

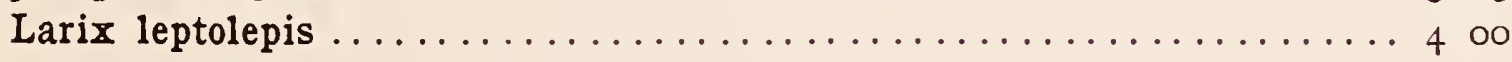

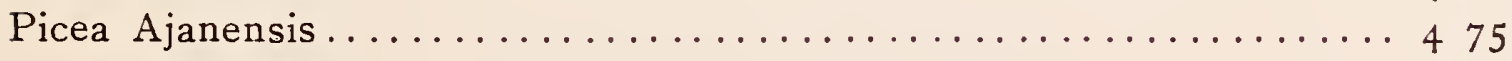

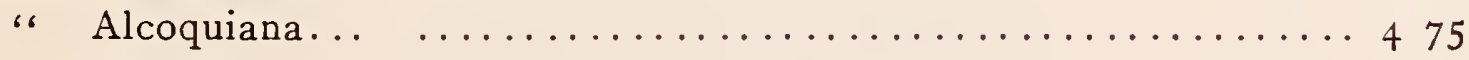

“ Glehni.............................. 8 o

" ${ }^{\prime}$ polita............................... 450

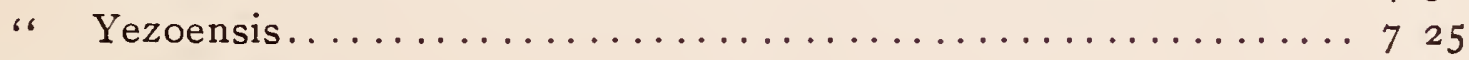

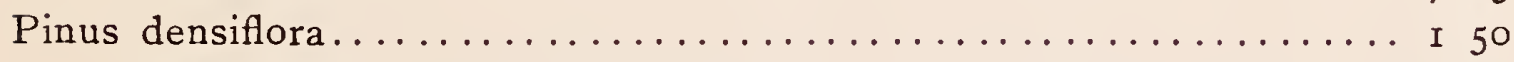

" Massoniana.......................... I oo

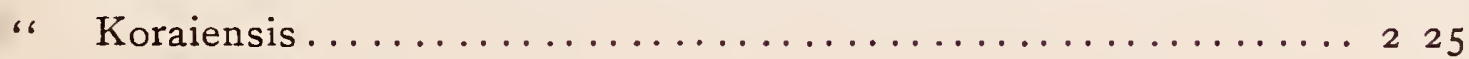

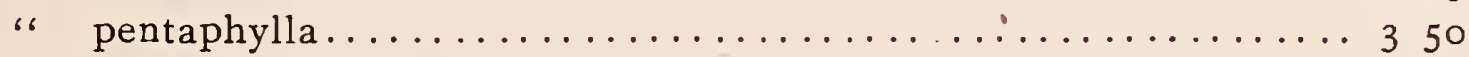

Podocarpus macrophylla....................... I 25

Sciadopitys verticillata.............................. 4 o

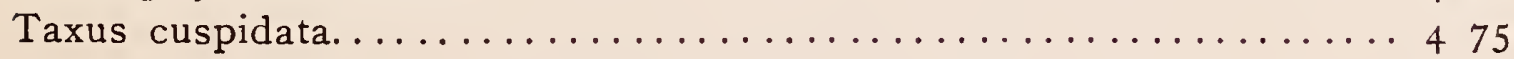


Thujopsis dolobrata.........................\$6 50

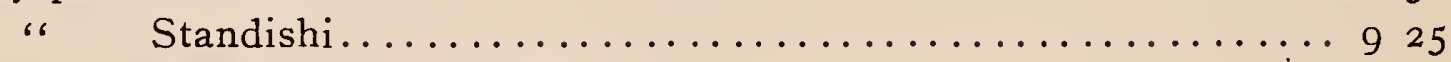

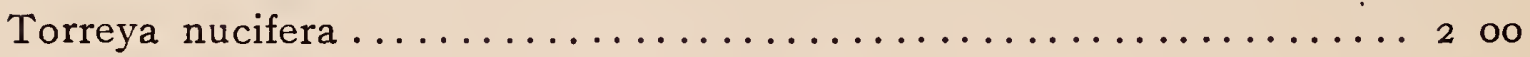

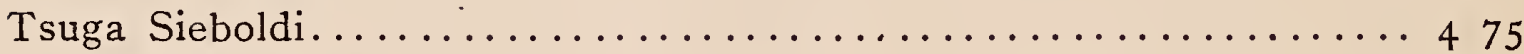

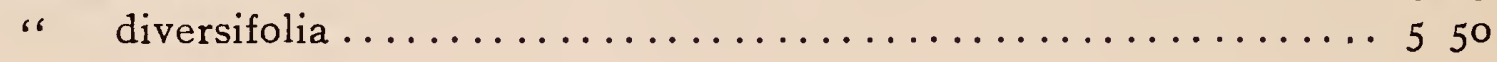

\section{Palm Seed}

Cycas revoluta.........................\$20 oo $\${ }_{16} 6_{5}$ oo

Livistona Chinensis .................... 8 oо 66 о

\section{Flower Seed}

Chrysanthemum seeds, selected from the choicest varieties, uncleaned

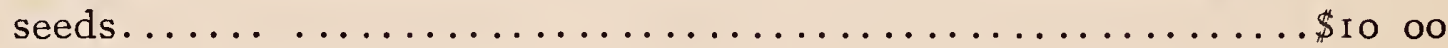

Euryale ferox (aquatic plant)

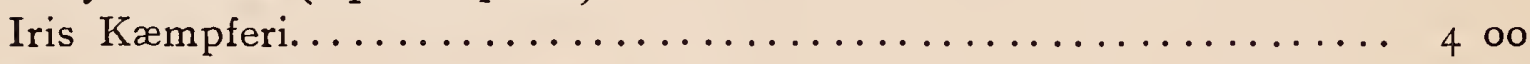

" Sibirica, var. orientalis..................... 350

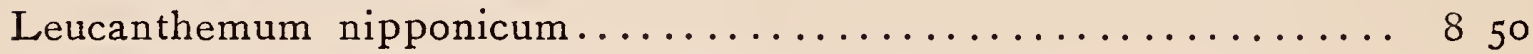

Nelumbium speciosum (Lotus), White................ I 50

“ speciosum (Lotus), Pink ................. I 50

Pæonia Moutan (Tree Peonies)..................... 325

" officinalis (Herbaceous Peonies)................. 3 oo

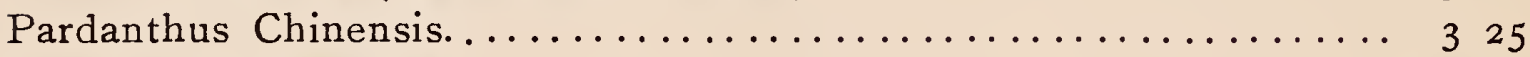

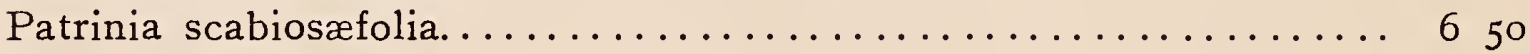

Physalis Francheti.......................... 6 о o

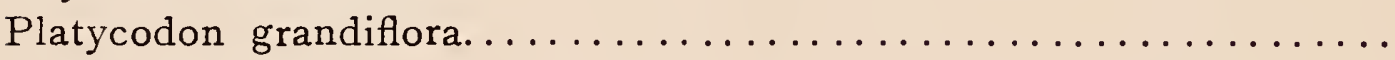

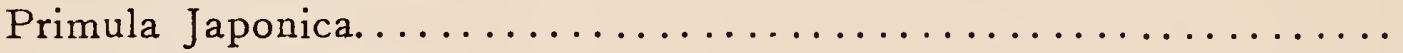

Pueraria Thunbergiana ......................... 6 oo

Scabiosa Japonica........................... $85_{50}$

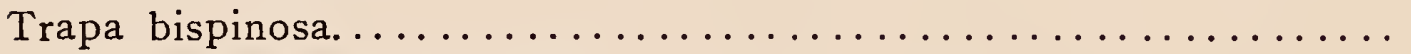

Trichosanthes cucumeroides....................... 2 oo

\section{Vegetable Seed}

Cucumber, Japanese Climbing.............per ro lbs., \$I6.50.. 2 oo

Eggplants, round or long....................... 75

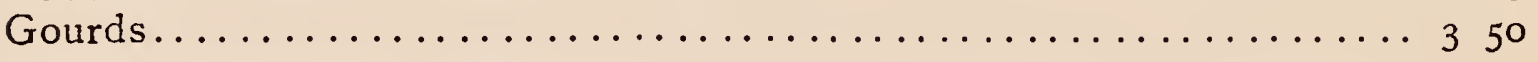

Radish, Sakurajima Mammoth. The largest Radish in existence.

Grows about 3 feet in circumference and weighs from 20

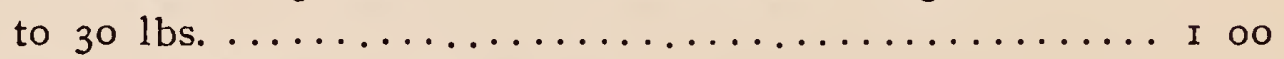

“ Long White Mikado (new variety)................ 6o

Soy Beans. Three varieties............................... 25

Turnip, Tennoji. Flat, about 8 inches in diameter........... I oo 


\section{Forage Plants}

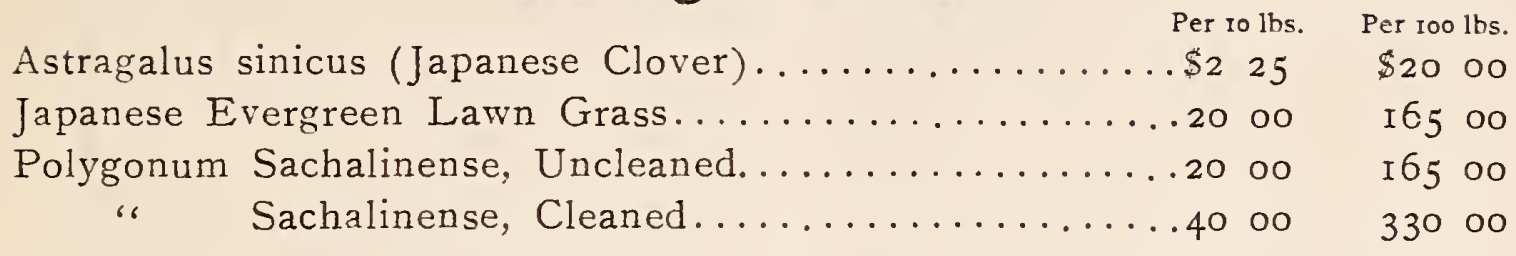

\section{List of Colored Hand Paintings}

Azalea flowers.................. per set of 25 varieties, $\$ 2$ 50

Bamboo plants ................. " "

Camellia flowers............... " " " 25 " 250

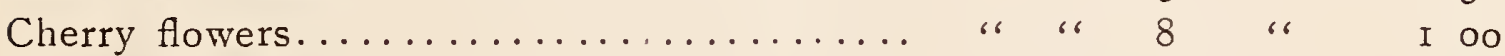

Chrysanthemums.................. " " " 100 " 725

Diospyros Kaki.................. " " "

Hydrangea flowers............... " " " 9 " 9 " 0

Iris flowers.................... " "

Lotus flowers.................. " " "

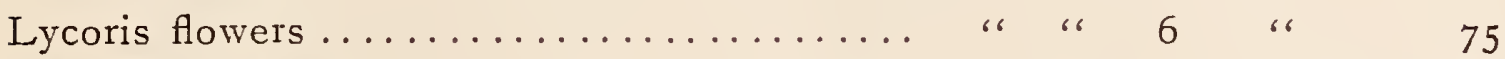

Magnolia flowers................. " " " 9 " $9 \ldots \ldots \ldots$

Orchids ...................." " 20 " 200

Plum fruit ................ " " $8 \ldots \ldots$ " $8 \ldots \ldots$

Pæonia, Tree................ " " " $50 \ldots \ldots$ " 325

" Herbaceous ............... " " 28 " " 2800

Lily flower book, lithographed ........ " " " " 36 " " 36 "

Maple leaves, lithographed .......... " " 39 " 39 "

Condor's Japanese Landscape Gardening

2 volumes, illustrated, $\$ 16.50$ 


\section{FERN BALLS}

\section{(Daballia bullata)}

Decidedly one of the most striking and best-paying novelties of recent years; of easy growth, not requiring much care, and occupying none of the space in the greenhouses required for other plants, being suspended from the rafters. In the fall the leaves die off, and the plants need a rest for one or two months, during which time we advise keeping them out of doors. The frost does not hurt the plants, as these Ferns are found on

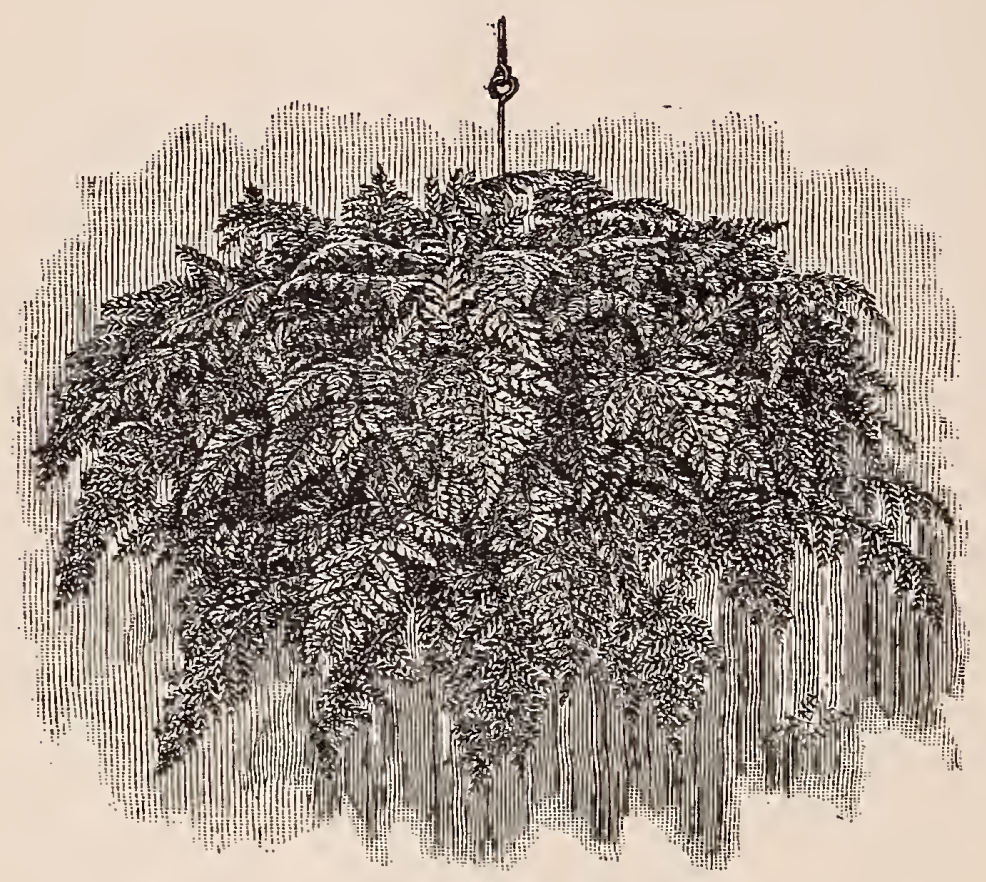

the Fuji Mountain, where they are exposed to the frost the entire winter. If in a frozen condition, they should not be taken into a warm room immediately, but allowed to gradually thaw out. The second year they will quickly bring out new leaves of a greater perfection than the first year.

We beg to state we handle only the "Nagaha," or long-leaved variety, the common variety producing only short fronds. We deliver these plants from January to April.

5 inches.......... . . $\$ 150$ per 1,000

7 to 9 inches .... $\$ 250$ per $1,000, \$ 2,000$ per 10,000 


\section{5}

(r)

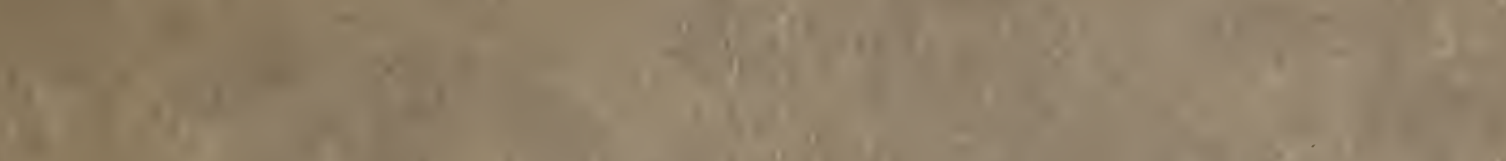

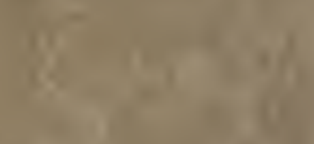
$\cos ^{3} x^{2}-x$

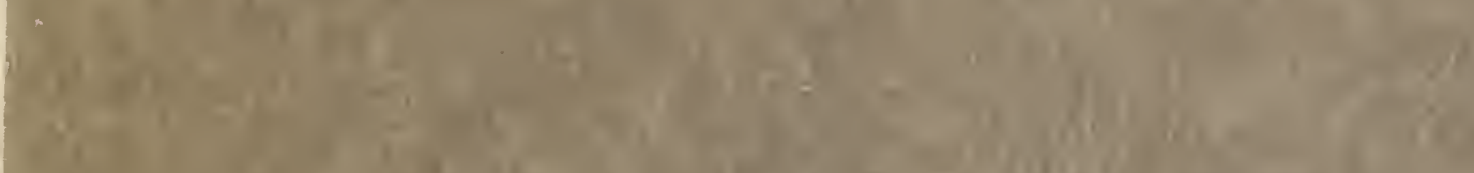

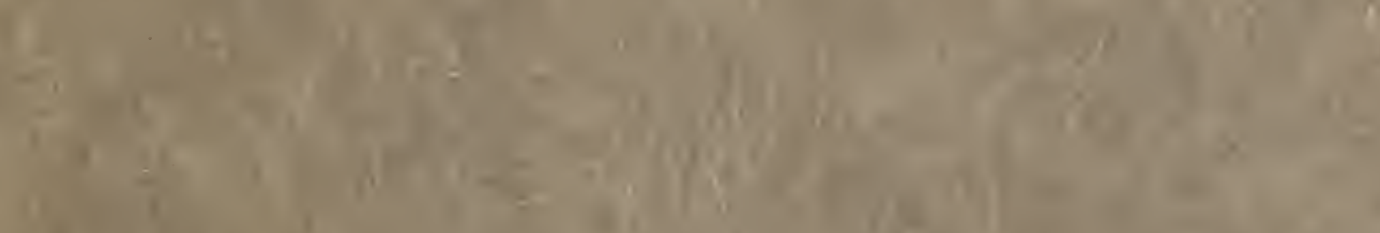

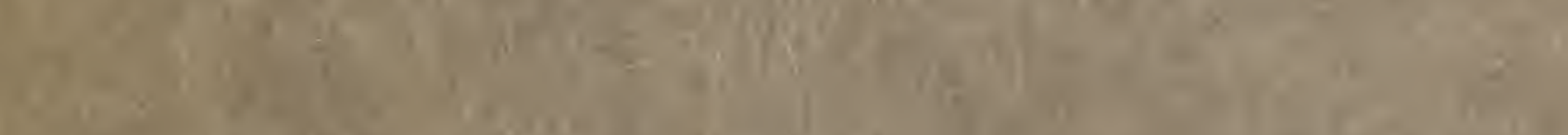

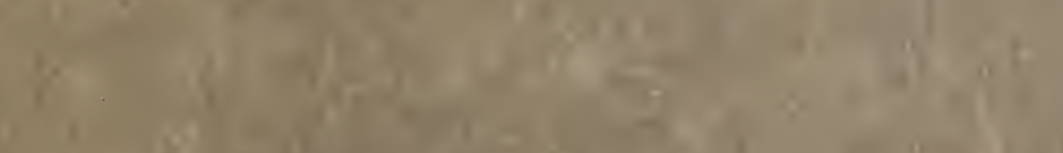

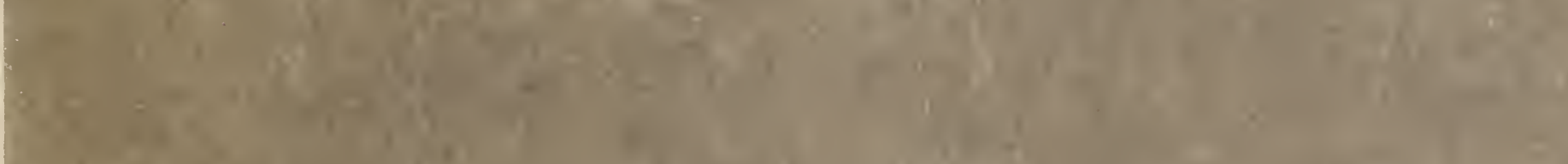
$2-50.6$

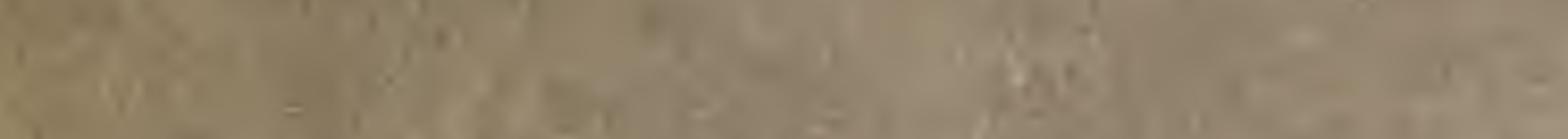

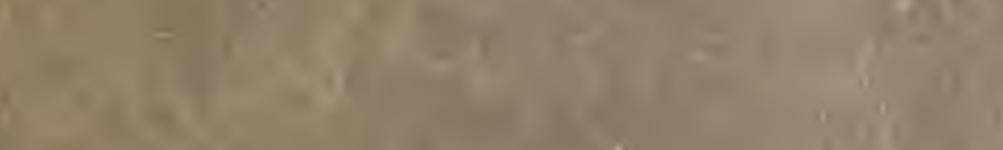

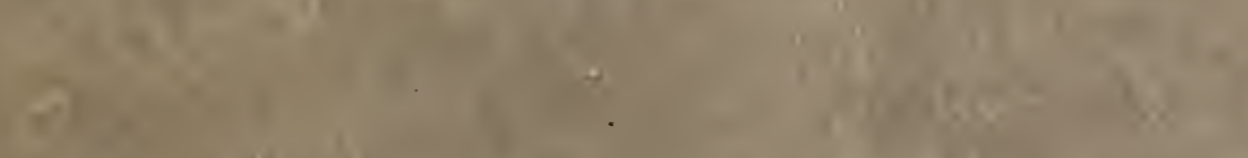

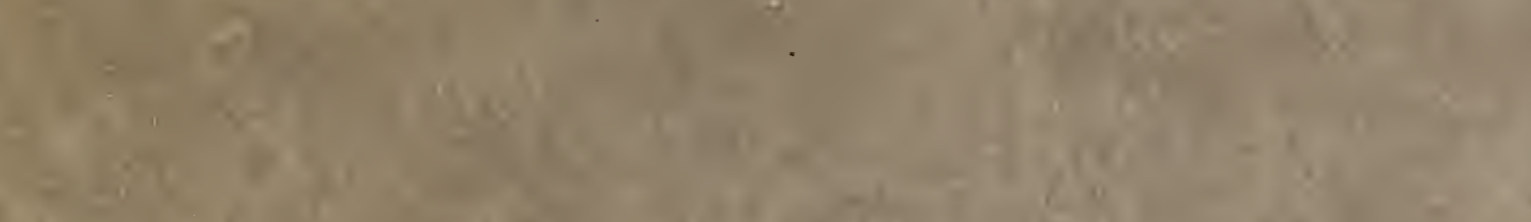

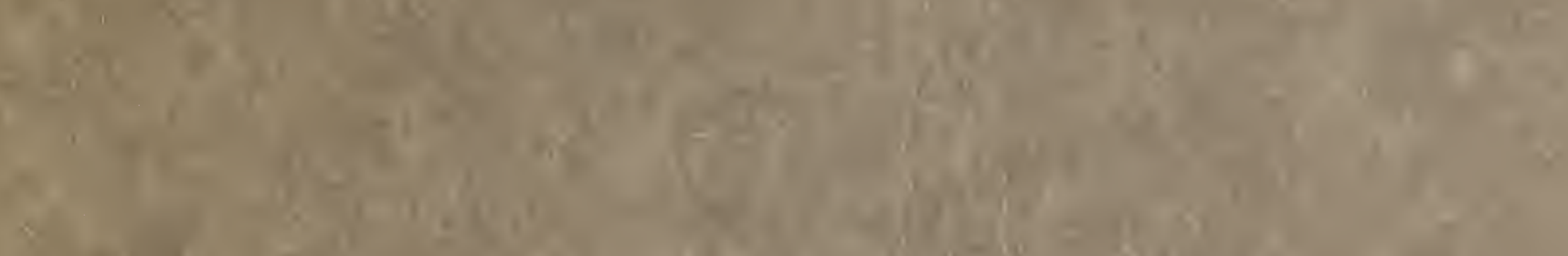

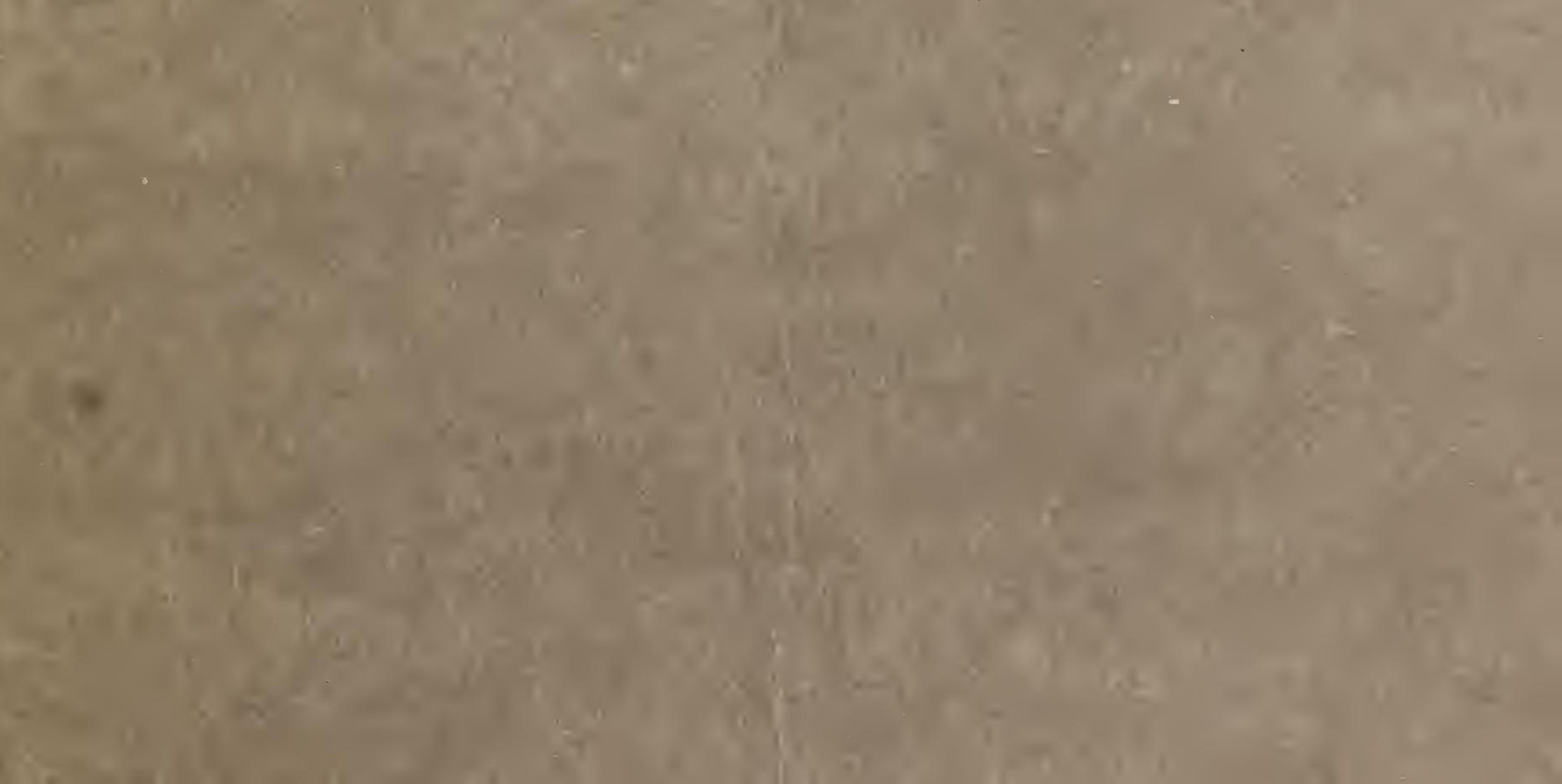




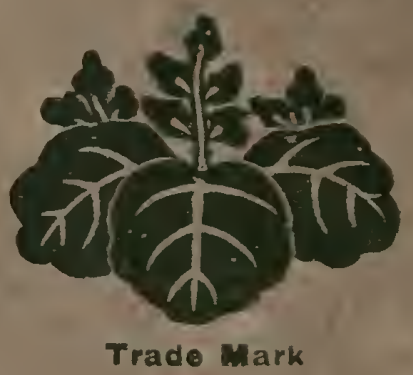

SUZUKI \& IIDA

31 Barclay St., New York 